INRA Prod. Anim.,

2012, 25 (2), 193-210

\title{
Productivité du travail et économie en élevages d'herbivores : définition des concepts, analyse et enjeux
}

\author{
T. CHARROIN ${ }^{1}$, P. VEYSSET ${ }^{2,3}$, S. DEVIENNE 4 , J.-L. FROMONT ${ }^{5}$, R. PALAZON ${ }^{1}$, M. FERRAND 6
}

${ }^{1}$ Institut de l'Elevage, BP 50, F-42270 Saint-Priest-en-Jarez, France

2 INRA, UMR1213 Herbivores, F-63122 Saint-Genès-Champanelle, France

${ }^{3}$ Clermont Université, VetAgro Sup, UMR Herbivores, BP 10448, F-63000 Clermont-Ferrand, France

${ }^{4}$ UFR Agriculture comparée et développement agricole, AgroParisTech, 16 rue Claude Bernard, F-75231 Paris,

France

5 AFOCG de l'Ain, 1 rue Robert Schuman, F-01000 Bourg-en-Bresse, France

${ }^{6}$ Institut de l'Elevage, 149 rue de Bercy, F-75595 Paris, France

Courriel :Thierry.Charroin@idele.fr

$\mathrm{Au}$ cours des cinquante dernières années, la productivité du travail s'est accrue en agriculture à un rythme beaucoup plus rapide que dans les autres secteurs économiques. Les agriculteurs ont adapté leurs structures et systèmes de production en utilisant plus d'intrants et de capitaux pour accroître la productivité de leur travail avec pour objectif de maintenir leur revenu. Pourtant, la relation entre volume de production par travailleur et revenu des éleveurs n'est pas linéaire.

La France a très longtemps été un pays rural dont la majorité des habitants vivait de l'agriculture. Alors que la population active agricole, familiale et salariée, représentait avec 6,2 millions de personnes $31 \%$ de l'emploi total en France en 1955 (Desriers 2007), cette part est tombée à $3,4 \%$ en 2008 (INSEE, enquête emploi). Dans le même temps, l'agriculture française connaissait une croissance de sa production tout à fait remarquable, soutenue par les lois d'orientation agricole de 1960 et 1962 qui ont impulsé de fortes et rapides évolutions des structures et dont l'effet a été renforcé par les premières organisations communes de marché (Politique Agricole Commune (PAC) issue du traité de Rome de 1957).

A partir des années 1980, le marché européen étant devenu excédentaire pour bon nombre de produits agricoles, différentes réformes de la PAC se sont succédé pour réguler l'offre tout en continuant dans un premier temps à soutenir les prix à la production, puis en passant à un soutien direct au revenu des agriculteurs (Butault 2004).

La tendance à la réduction du nombre d'exploitations et de travailleurs agricoles se poursuit encore aujourd'hui. Le recensement agricole 2010 (Giroux 2011) montre que le nombre total de travailleurs agricoles a chuté de $22 \%$ entre 2000 et 2010 , le nombre d'exploitations diminuant de $26 \%$ (en 2010 les 490000 exploitations agricoles que compte la France métropolitaine emploient 750000 Unités de Travail Annuel (UTA)). La superficie agricole totale poursuit également son érosion (- $3 \%$ sur dix ans). $\mathrm{Au}$ final, la surface que valorise en moyenne un travailleur agricole s'est accrue de $24 \%$ depuis 2000 (36 ha/travailleur en 2010 contre 29 ha/travailleur en 2000) (Desriers 2011).

Le nombre d'exploitations d'élevage a diminué et les cheptels ont connu des évolutions contrastées selon les périodes et les espèces. Le nombre total de bovins s'est accru entre 1950 et 1980 (passant de 15,8 millions de têtes à 23,1 millions soit près de $50 \%$ d'augmentation), avant de régresser de $17 \%$ jusqu'aux années 2000 (20,5 millions de bovins en 2000, 19,1 millions en 2011) sous l'effet des quotas laitiers et des Primes au Maintien du Troupeau de Vaches Allaitantes (PMTVA). La PAC a aidé à «fixer» les productions d'herbivores dans l'ensemble des régions, bien qu'on assiste à une légère concentration des bovins laitiers dans l'Ouest et des bovins à viande dans le Massif Central (Institut de 1'Elevage 2011a). La tendance est toujours à la croissance de la taille moyenne des troupeaux : $+38 \%$ entre 2000 et 2010 pour le nombre moyen de vaches laitières par exploitation et $+31 \%$ pour celui de vaches allaitantes, même si le travail est souvent évoqué comme un facteur limitant par beaucoup d'éleveurs.

Parallèlement, le nombre d'élevages hors sol et les effectifs de porcs et volailles ont explosé. Ces élevages, non soutenus par la PAC, se sont concentrés en Bretagne et Pays de la Loire (Bourgeois et Desriers 2002), mouvement lié à la forte intégration de la filière par des firmes agro-alimentaires qui cherchent à limiter leurs coûts grâce à des économies d'échelle et à un accès facile à une production standardisée (Bagoulla et al 2010).

Les agriculteurs se sont ainsi toujours adaptés (structures, systèmes de production, pratiques) au contexte économique et politique en privilégiant avant tout l'augmentation de la productivité de leur travail. En cinquante ans, les gains de productivité en agriculture ont été continus et considérables. En parallèle, les prix agricoles à la production 
ont fortement baissé, compensés en partie et depuis vingt ans seulement par des soutiens directs. Cette baisse a surtout profité aux branches clientes de l'agriculture, notamment aux industries agro-alimentaires (la France est devenue un grand exportateur de produits agro-alimentaires). A contrario, les consommateurs en ont peu bénéficié, sauf pour les produits de l'industrie de la viande et du lait : le taux de marge du commerce a plutôt baissé de 1979 à 1994, mais il augmente fortement depuis, entraînant un accroissement en termes réels des prix à la consommation (Butault 2008).

Nous débuterons cet article en précisant dans un premier temps la notion de main-d'œuvre en exploitation agricole, ainsi que les différentes définitions de la productivité physique et économique du travail. Nous introduirons également les principaux indicateurs utilisés pour juger de la performance économique des exploitations.

Nous porterons ensuite un regard sur l'évolution de la productivité du travail et de l'économie de la production agricole française au cours des 50 dernières années. Un zoom sur les trajectoires d'exploitations d'élevage bovin allaitant pendant 20 ans illustrera ces tendances et précisera leurs impacts productifs et économiques. Enfin, nous analyserons la relation entre productivité du travail et résultats économiques en élevage, pour les différents types d'élevages herbivores (bovins, ovins, caprins pour l'année 2009) et ouvrirons le débat sur la recherche de gains de productivité en élevage, leurs moteurs, leurs intérêts et leurs limites.

\section{1 / Productivités du travail et indicateurs économi- ques : concepts et défini- tions}

\section{1 / Quantification de la main- d'œuvre}

Il est assez difficile de quantifier la main-d'œuvre en agriculture du fait de l'importance de la main-d'œuvre non salariée dont le nombre d'heures de travail est rarement enregistré. On a donc le plus souvent recours à des conventions.

Afin d'analyser l'organisation du travail, comme le propose la méthode du Bilan Travail (INRA et l'Institut de l'Elevage, Dedieu et al 2000), nous avons distingué trois classes pour la main-d'œuvre : «exploitants et associés», «salariée» et «bénévole».
Pour sa quantification, nous comptabilisons une unité de main-d'œuvre pour toute personne majeure «à temps plein» sur l'exploitation, quel que soit son âge (même si son temps réel de travail dépasse largement les normes en vigueur pour le travail salarié). Pour les temps partiels (représentations professionnelles importantes, autres activités pour l'exploitant ou son conjoint, coup de main bénévole) nous disposons de normes qui, lors des suivis en exploitations, sont ajustées par échange avec les éleveurs. Cette démarche rejoint la pratique du Service de la Statistique et de la Prospective (SSP) (Lacroix et Mollard 1991).

Le vocable «Unité de Main-d'œuvre» (UMO) a été préféré à celui «d'Unité de Travail Annuel» (UTA) qui faisait référence par le passé à un nombre d'heures (degré de précision difficile à approcher).

\section{2 / Productivités du travail}

La productivité du travail peut être définie de deux manières : la «productivité physique» qui mesure le volume de biens produit par UMO (unités physiques de lait ou de viande produit en exploitation d'élevage) et la «productivité économique» qui ambitionne de quantifier la richesse créée par UMO. Leur analyse sur un intervalle de temps permet de mesurer des gains de productivité. Nous allons préciser pour chacune d'entre elles les conventions méthodologiques que nous avons retenues pour cette analyse.

\section{a) Productivité économique du travail}

La productivité économique du travail correspond à la richesse créée par les actifs ayant participé au processus de production ; elle est mesurée par la valeur ajoutée, brute ou nette, par actif agricole. Celle-ci est égale à la valeur de la production (hors aides) diminuée de celle de l'ensemble des biens et services produits par des tiers (consommations intermédiaires et consommation annuelle moyenne de capital fixe). Pour la valeur ajoutée brute, la dépréciation du capital fixe n'est pas soustraite; on passe à la valeur ajoutée nette en déduisant cette consommation. Ces grandeurs économiques peuvent être calculées au niveau de l'exploitation, mais aussi au niveau de la branche agricole dans les Comptes de la Nation. Notons que la valeur ajoutée peut varier significativement dans le temps en fonction de la conjoncture économique de l'année et indépendamment de la productivité physique.

Pour s'affranchir des effets de la conjoncture et estimer in fine la crois- sance réelle de la richesse produite, les Comptes de la Nation «décomposent les évolutions des opérations sur les biens et services en valeur en deux composantes : un facteur prix, reflétant le mouvement des prix, et un facteur volume, mesurant l'évolution des «quantités» des agrégats (produit brut, consommations intermédiaires, valeur ajoutée...) à prix constants» (Braibant 2007). Cette décomposition s'effectue produit par produit, en tenant compte de la déformation des prix relatifs.

\section{b) Productivité physique du travail}

Les notions de production rapportée à des unités non monétaires telles la productivité numérique (nombre de produits par femelle reproductrice), pondérale ( $\mathrm{kg}$ de viande vive par Unité Gros Bétail (UGB)), fourragère (tonnes de matière sèche par ha), etc. sont aujourd'hui bien intégrées. En complément, il est de plus en plus fréquemment fait référence à la productivité physique du travail. Cet indicateur est relativement aisé à appréhender dans les exploitations ayant un seul atelier de production. Pour les exploitations d'élevage avec plusieurs ateliers de production (polyculture-élevage, élevage diversifié...), certains auteurs ont proposé des méthodes pour quantifier la productivité physique globale de l'exploitation (Benoit et Laignel 2006) en convertissant des hectares de grandes cultures, des marges brutes d'atelier hors sol en «équivalent UGB» pour les cumuler aux UGB de l'atelier animal et présenter ainsi un indicateur. Mais de fait, ces conventions évaluent une dimension et non pas une productivité (qui peut être très différente).

Devant l'absence d'une méthodologie partagée par tous les acteurs (recherche, développement, enseignement, agriculteurs...), notre approche s'est refusée à cumuler des quantités de nature aussi différente que par exemple le lait et la viande.

Nous avons préféré désagréger la main-d'œuvre totale de l'exploitation au niveau de l'atelier. Un jeu de coefficients d'affectation déterminés par régression multiple dans une étude précédente (Charroin et Ferrand 2010) nous permet de calculer la part de maind'œuvre affectée à chaque atelier (celles nécessaires au troupeau, aux surfaces fourragères et aux céréales intraconsommées) et donc à en évaluer la productivité physique. Pour les élevages d'herbivores, le volume de production correspond au produit principal de la filière : litres de lait pour les productions laitières et kilos de viande vive (calculée à partir des ventes et achats d'animaux et des variations d'inventaire 
Encadré 1. Schéma des soldes intermédiaires de gestion.

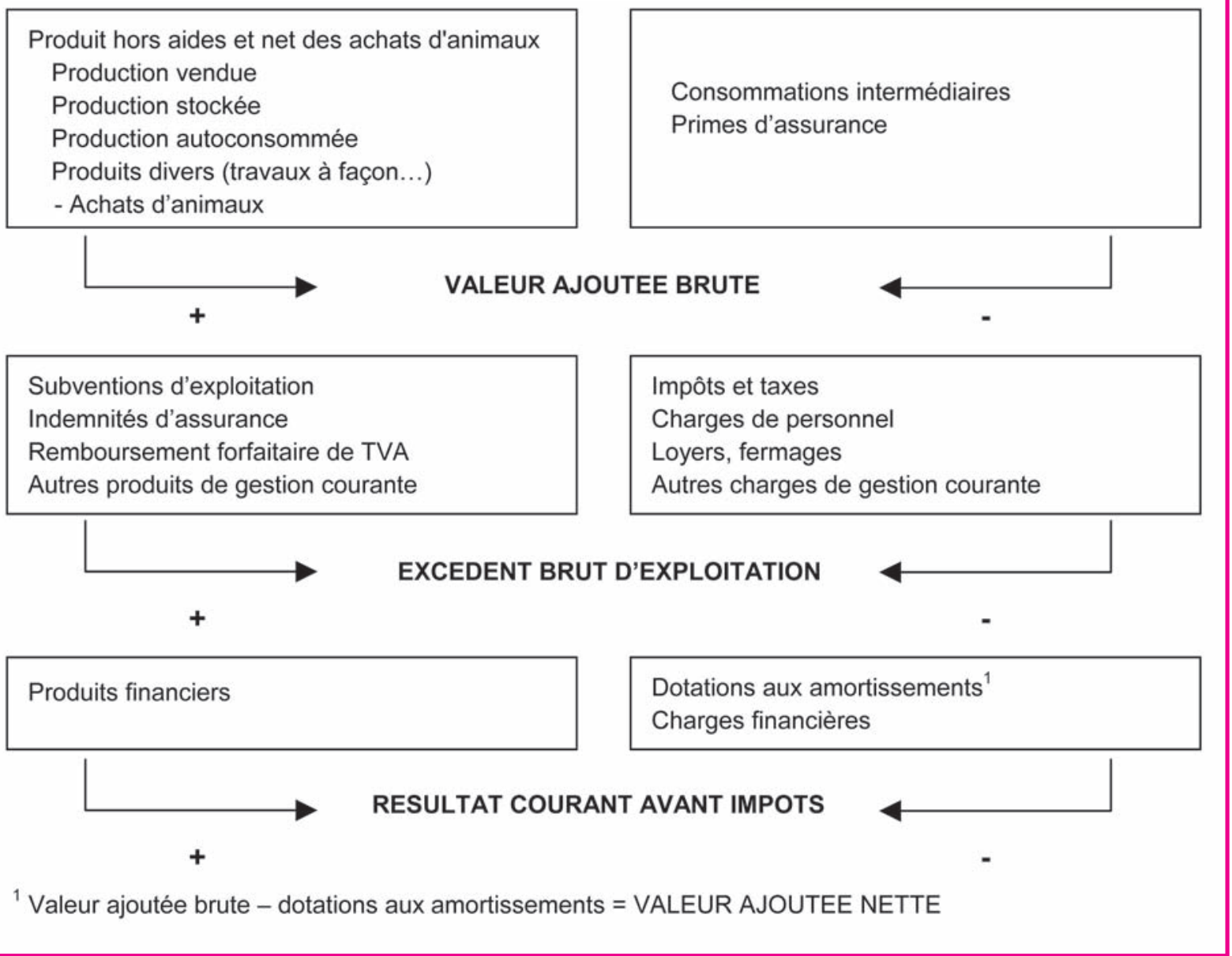

en nombre et en poids) pour la viande bovine. En viande ovine, en raison de la forte différence de valorisation entre agneaux et brebis de réforme et de la prépondérance des agneaux vendus en carcasse, le volume de production est exprimé en «équivalents agneaux carcasse». Nous avons renoncé à prendre en compte les coproduits (veaux de 8 jours pour les troupeaux laitiers, laine en élevage ovin...) après avoir vérifié leur faible poids dans le chiffre d'affaires.

Pour tous les ateliers herbivores de chaque exploitation de la base de données des Réseaux d'élevage, nous disposons des résultats de productivité physique par UMO rémunérée (une évaluation de l'atelier «grandes cultures» est en cours). Ce mode d'estimation de la productivité physique du travail, maintenant intégré dans les outils «coûts de production», a été systématisé dans toutes les filières herbivores.

\section{3 / Indicateurs économiques}

L'impact de la productivité du travail est analysé selon les grandes composantes du compte d'exploitation : produit brut avec et sans les aides, contribution des aides et part des intrants. Ce dernier poste (concentrés, fourrages et paille achetés, engrais, semences, produits de protection phytosanitaire, carburants, électricité et gaz) regroupe les charges les plus liées au degré d'intensification des systèmes de production et donc a priori à un accroissement de la productivité.

Parmi les soldes intermédiaires de gestion (encadré 1), nous avons retenu la valeur ajoutée brute calculée sans aide, la valeur ajoutée nette après déduction des amortissements, l'Excédent Brut d'Exploitation (EBE), ainsi que le Résultat Courant Avant Impôts (RCAI) qui est l'indicateur du revenu des comptes de l'agriculture. Afin de pouvoir analyser la productivité du travail de la main-d'œuvre rémunérée (salariée ou non), le RCAI et l'EBE sont calculés sans déduire les salaires, les charges salariales et les charges sociales de l'exploitant. L'efficience économique des exploitations est analysée à partir du ratio EBE/Produit brut total. Le montant du capital d'exploitation est calculé hors foncier (du fait de la dimension patrimoniale que celui-ci représente).

La rétrospective de l'évolution de la productivité du travail de l'agriculture française sur les 50 dernières années fera référence aux critères utilisés par les Comptes de la Nation. Les données disponibles pour traiter du zoom sur les trajectoires d'exploitations bovines allaitantes et les analyses des relations entre la productivité du travail et les résultats économiques feront référence à la productivité physique du travail.

\section{2 / Evolution de la producti- vité du travail et des résul- tats économiques de l'agri- culture française depuis 1950}

Depuis cinquante ans, la production agricole française a connu, nous l'avons vu, une forte expansion malgré la baisse importante du nombre d'exploitations et d'actifs agricoles. Cette évolution repose sur un accroissement spectaculaire du volume produit par 
actif agricole, donc de la productivité physique du travail, permis par de profondes transformations des systèmes de production agricole ${ }^{1}$.

\section{1 / Les systèmes de polycultu- re-élevage des années 1950}

En 1950, dans la majeure partie des régions françaises, prédominaient des systèmes de production basés sur l'association agriculture-élevage, très largement tournés vers l'autoconsommation familiale (Mazoyer et Roudart 1997). Ces systèmes combinaient différents systèmes de culture (adaptés à la diversité des terroirs) et d'élevage qui entretenaient des relations étroites. Les systèmes de culture étaient généralement basés sur des rotations complexes qui faisaient alterner céréales, plantes sarclées (betteraves fourragères et/ou à sucre, pommes de terre, navets, choux...) et légumineuses fourragères (trèfle semé sous avoine, luzerne, sainfoin...). Les produits végétaux fournissaient une part importante de l'alimentation de la famille, ainsi que l'essentiel de l'alimentation des élevages. La logique agronomique des rotations de cultures était basée sur les effets «précédent» et «sensibilité du suivant»: les légumineuses contribuaient à enrichir le sol en azote tandis que l'alternance des plantes sarclées et des prairies temporaires ou artificielles avec les céréales, contribuait à améliorer la structure du sol, à limiter l'impact des maladies et à contrôler la prolifération des adventices et des prédateurs. Un travail manuel important était encore nécessaire pour le désherbage et les rendements demeuraient limités.

Les productions fourragères ainsi que les sous-produits de culture contribuaient au fonctionnement des systèmes d'élevage. Les exploitations élevaient bien souvent à la fois des bovins, pour la production de lait et de viande, des ovins et/ou des caprins dans les régions aux conditions de milieu plus difficiles et, à l'exception des plus petites d'entre elles, des chevaux pour la force de trait, ainsi que quelques porcs (porcs à l'engrais et parfois une ou quelques truies) et de la volaille. Ces différents systèmes d'élevage fournissaient en retour l'énergie de traction et le fumier pour la reproduction de la fertilité sur les espaces cultivés. Le système de polyculture-élevage reposait ainsi sur une large autofourniture des moyens de production.

L'autoconsommation et l'autofourniture absorbaient en général la majeure partie de la production agricole et les surplus étaient vendus, parfois après transformation, afin de dégager le revenu monétaire nécessaire pour renouveler les moyens de production de l'exploitation (notamment l'équipement de culture attelée) et assurer les besoins de la famille.

\section{2 / Une révolution agricole basée sur l'incorporation de moyens de production indus- triels}

A partir des années 1950, une véritable révolution agricole (Mazoyer et Roudart 1997) a pris place et s'est déroulée en plusieurs phases jusqu'à aujourd'hui. Elle a reposé sur l'utilisation croissante de moyens de production industriels et sur le développement de techniques conçues par la recherche publique ou privée et vulgarisées dans les centres de formation ou directement auprès des agriculteurs.

Les innovations techniques, sur lesquelles repose l'accroissement de la productivité du travail dans le secteur agricole, ne sont pas conçues indépendamment du système technique et de l'esprit technologique ambiant (Mounier 1992, Perez 2009). Ainsi, depuis 1950 , les solutions proposées et majoritairement adoptées dans le secteur agricole se sont fondées dans un premier temps sur le pétrole (industrie du tracteur et du machinisme agricole, pétrochimie...), une division horizontale et verticale du travail et des économies d'échelle, la standardisation des productions, etc. avant que l'informatique et les biotechnologies ne prennent récemment une place grandissante. La biologie a été mise au service du développement de ces technologies. La sélection génétique a visé à adapter les plantes cultivées et les animaux aux moyens de production industriels, tandis que les écosystèmes, considérés seulement comme un support de production, ont été aménagés afin de permettre la mise en œuvre des nouvelles formes de production (arrachage des haies, agrandissement des parcelles, drainage, irrigation...). Le contrôle des cycles de matière (eau, azote, phosphore, oligoéléments...) et des populations vivantes a été extrêmement poussé. Le mode de production développé ne s'est que peu appuyé sur les capacités intrinsèques des écosystèmes à produire et à se renouveler, privilégiant au contraire le recours aux moyens de production industriels.

L'adoption d'équipements de plus en plus performants a permis aux exploitants agricoles d'accroître l'efficacité de leur travail et de toujours repousser la limite du nombre d'hectares ou d'animaux qu'un actif peut prendre en charge. Le recours aux intrants a contribué à accroître les rendements, à simplifier le travail. Il a également permis d'abandonner la complémentarité jusqu'alors indispensable entre les cultures au sein des rotations et entre les systèmes de culture et d'élevage. Le processus de remplacement de l'autofourniture des moyens de production par l'approvisionnement auprès de l'industrie a ouvert la voie à la spécialisation des unités de production dans un nombre de plus en plus restreint de productions végétales et/ou animales, en fonction des conditions de milieu, de leur superficie et de leurs moyens de production ainsi que des débouchés auxquels elles avaient accès. Aujourd'hui seules $12,6 \%$ des exploitations agricoles françaises sont en polyculture ou polyélevage, contre $16,8 \%$ en 1988 ; Sébillotte (1966) estimait qu'elles étaient $85 \%$ en 1963. L'accroissement de la productivité physique du travail s'est également accompagné d'un agrandissement de la superficie et de la taille de troupeau des exploitations, permis par l'adoption de nouveaux équipements et nécessaire pour les rentabiliser. Ce processus a été rendu possible par la disparition progressive et continue des exploitations insuffisamment productives pour suivre ce mouvement ; il aboutit à une concentration de la production dans un nombre toujours plus restreint d'exploitations.

\section{3 / Des systèmes de production spécialisés de plus en plus pro- ductifs}

En grandes cultures, le développement a reposé sur une spécialisation de plus en plus poussée des systèmes de production et sur la simplification et

\footnotetext{
${ }^{1}$ La description des modalités concrètes d'évolution des systèmes de production s'appuie sur les nombreux travaux de terrain réalisés dans différentes régions françaises depuis 20 ans visant à effectuer l'analyse-diagnostic de l'agriculture de petites régions agricoles (Cochet et Devienne 2006). Ces travaux, encadrés par les enseignants de l'UFR d'Agriculture comparée et Développement agricole d'AgroParisTech, ont été effectués soit dans le cadre de mémoires d'Ingénieur en Développement Agricole ou lors de travaux de terrain collectifs avec des étudiants de la dominante d'approfondissement Développement agricole ou en 2 ème année du cursus agronome d'AgroParisTech. Au total, plus de cinquante diagnostics ont été réalisés, dans des régions très différentes (Nord, Haute Marne, Meuse, Côtes d'Armor, Vendée, Deux Sèvres, Cantal, Yonne, Corrèze, Drôme, Seine Maritime, Orne, Charente, Allier...). Ces diagnostics visent à comprendre la situation actuelle et l'évolution de l'agriculture à l'échelle d'une petite région agricole en mettant en évidence le processus de différenciation des systèmes de production qui a conduit à la situation actuelle.
} 
le raccourcissement des rotations. L'accroissement de la production par actif a été permis par l'augmentation des rendements et de la superficie. L'utilisation de variétés sélectionnées, des engrais et des produits phytosanitaires et, dans bien des cas aussi, le drainage, voire l'irrigation, ont conduit à des rendements accrus (qui sont passés par exemple pour le blé de $25 \mathrm{q} / \mathrm{ha}$ en 1960 à 70 q au début des années 2000 et plafonnent depuis). La superficie cultivée par actif a pu être augmentée non seulement grâce à l'adoption d'équipements autorisant une plus grande capacité de travail (puissance et largeur de travail), mais aussi grâce à la simplification des itinéraires techniques (travail simplifié, semis direct) que permet le recours aux herbicides. Cette évolution devrait se poursuivre avec le développement de l'informatique et des biotechnologies. Le recours au GPS permet en effet à la fois l'autoguidage des machines, donc un allègement substantiel du travail ${ }^{2}$ et la possibilité, avec les mêmes puissance et largeur de travail, d'accroître la superficie maximum cultivable par actif et une gestion précise de l'information sur des superficies importantes, donc l'ajustement précis de l'utilisation des intrants. Les biotechnologies quant à elles, si elles sont autorisées comme aux EtatsUnis, offrent avec les variétés génétiquement modifiées (tolérantes aux herbicides et/ou résistantes à certains insectes) la possibilité de simplifier le travail en réduisant encore le nombre de passages sur la parcelle.

L'accroissement de la productivité du travail en grandes cultures repose ainsi sur un capital fixe par actif de plus en plus élevé et la mise en œuvre d'itinéraires techniques s'appuyant sur un recours important aux consommations intermédiaires, en dépit des efforts réalisés au cours des vingt dernières années pour maîtriser l'utilisation des intrants.

Le même processus est à l'œuvre dans le secteur de l'élevage. En élevage bovin laitier, la voie privilégiée d'augmentation de la productivité du travail a consisté en l'augmentation du nombre de vaches laitières par actif et de la production laitière par vache. L'adoption de bâtiments permettant de réduire le temps de travail nécessaire à l'alimentation des animaux et à la manutention des déjections a été couplée avec celle d'équipements plus performants (fosses à lisier, désileuses-pailleuses puis mélangeuses distributrices tractées ou automotrices, robots d'alimentation, pots trayeurs dans les années 1950 jusqu'au robot de traite ou carrousel automatisé depuis les années 1990...). Dans les exploitations équipées de l'automatisation, la traite, tâche la plus contraignante pour les éleveurs laitiers, cesse d'être le facteur limitant du nombre de vaches par actif; la surveillance des chaleurs devient alors la principale contrainte et de nouvelles techniques ont été développées permettant de repousser cette limite. L'externalisation de certaines tâches, comme le recours à l'entreprise pour la réalisation des opérations culturales, permet par ailleurs de spécialiser le travail de l'exploitant ainsi que le capital d'exploitation et d'accrô̂tre le nombre de vaches laitières qu'il peut élever.

Parallèlement les éleveurs ont cherché, afin de rentabiliser les investissements coûteux qu'ils réalisaient dans les équipements, à augmenter les rendements laitiers (qui sont passés de 2370 L/vache en 1960 à 3100 en 1970 et 6340 en 2008). La sélection génétique a visé notamment à accroître le potentiel de production des vaches, tandis que le recours à des fourrages de densité énergétique plus élevée, distribués avec des concentrés riches en protéines comme le tourteau de soja acheté à l'extérieur, permettait à ce potentiel de s'exprimer. La production de ces fourrages est allée de pair avec une modification des systèmes fourragers visant à accroître les rendements et à augmenter le chargement. Ce mouvement a été réalisé en plusieurs étapes. Dans les années 19501960, la priorité a été donnée à la «révolution fourragère», basée sur le développement des prairies temporaires de raygrass anglais ou italien et sur l'introduction de la technique de l'ensilage, permettant à la fois de mieux tirer parti du pic de croissance de l'herbe au printemps et d'obtenir un fourrage beaucoup plus digestible que le foin. Les techniques de gestion de la prairie, notamment de pâturage tournant et de fertilisation, ont elles aussi été vulgarisées à cette époque (Dumont 1954, Voisin 1957, Pochon 2002, Pochon 2008).

A partir des années 1970, la culture de maïs ensilage commence à être développée en plaine ainsi que dans certaines régions de moyenne montagne. Elle présente l'avantage de permettre des rendements et une densité énergétique élevés, de bien se conjuguer avec des tourteaux riches en protéines pour l'équilibre de la ration et d'être entièrement méca- nisable, du semis à la distribution aux vaches (Guesdon 1985). Culture annuelle relativement coûteuse en intrants (semences, herbicides, engrais), le développement du maïs nécessite souvent des investissements importants : drainage de parcelles au potentiel de rendement élevé mais dont la portance du sol n'est pas suffisante à l'automne pour permettre la récolte ; irrigation dans les régions au déficit hydrique marqué en été afin d'assurer la régularité des rendements; construction de bâtiments munis de couloirs d'affouragement adaptés pour la distribution de l'ensilage. Les prairies cèdent progressivement la place au maïs : les prairies permanentes situées dans les espaces les plus favorables à la culture du maïs (fonds de vallée par exemple) sont retournées tandis que les prairies temporaires reculent dans les rotations, en dépit des améliorations apportées aux techniques de conservation de l'herbe (perfectionnement des techniques d'ensilage et développement de l'enrubannage à partir des années 1990). Ainsi, si depuis 1960 les cultures fourragères ont reculé de plus de 5 millions d'hectares, l'évolution de la superficie des différents types de fourrages a été contrastée : recul continu des superficies toujours en herbe (- 3,5 millions d'hectares), diminution brutale de la superficie des prairies artificielles dans les années 1960 et 1970 (passées de plus de 3 millions d'hectares en 1960 à moins d'un million en 1980 et un peu moins de 400000 ha en 2010), stabilité de la superficie des prairies temporaires depuis 1970, après une progression de près d'un million d'ha entre 1960 et 1970 ; quant au maïs fourrage, pratiquement absent des assolements dans les années 1960, il a vu sa superficie exploser à partir de 1970, pour atteindre 1,7 million d'hectares au début des années 1990 et se stabiliser ensuite autour de 1,5 million d'hectares.

Les systèmes fourragers qui se sont développés depuis les années 1970 sont, excepté en zone de montagne, de plus en plus dépendants du maïs. D'abord utilisé pour la seule ration hivernale, l'ensilage de maïs est progressivement donné en complément du pâturage pendant la période estivale, puis de plus en plus tôt au printemps jusqu'à des systèmes d'alimentation dans lesquels les silos sont ouverts toute l'année et procurent l'essentiel de l'alimentation des vaches, lesquelles ne prélèvent plus que très peu de fourrages à la pâture voire sont élevées en zéro-pâturage. Cette évolution a autorisé l'agrandisse-

\footnotetext{
2 Le recours à l'autoguidage, permis par le GPS («Global Positioning System») permet de supprimer le travail de conduite de la machine et de surveillance pour la réalisation de l'opération culturale, la machine effectuant seule le travail avec une précision de l'ordre de un ou deux centimètres.
} 
Figure 1. Evolution de la production agricole, des consommations intermédiaires et de quelques postes spécifiques en volume.

(1959 = indice 100). Source : Insee, Comptes de la Nation.

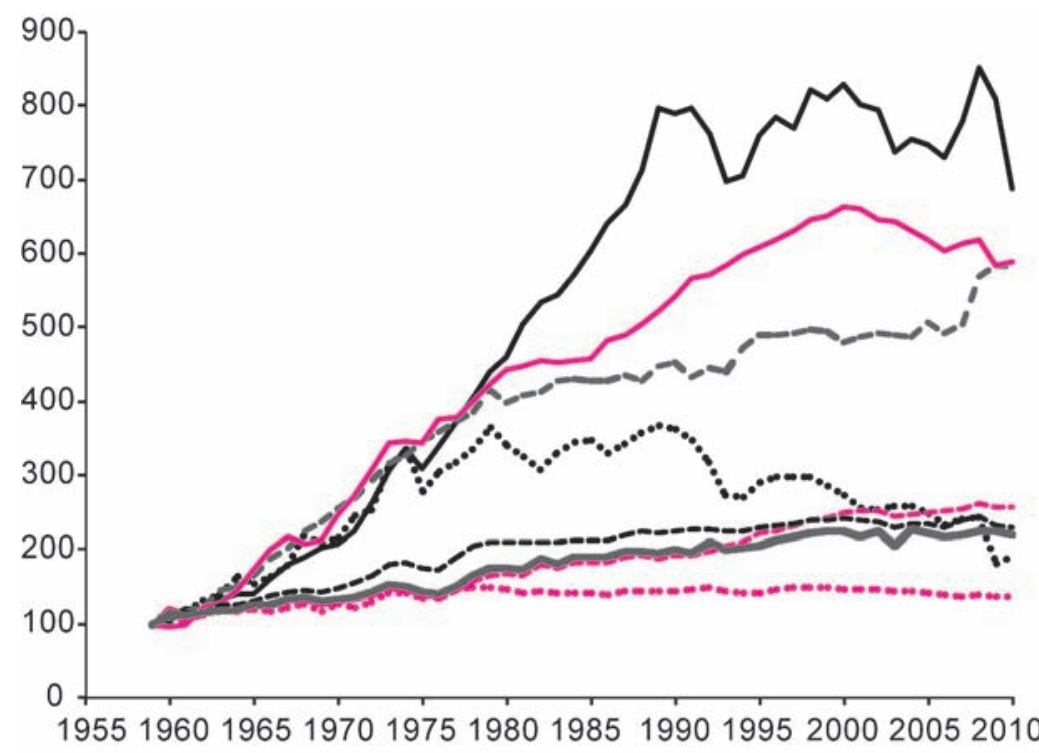

$\begin{array}{ll}\text {......... Energie et lubrifiants } & \begin{array}{l}\text { Aliments pour animaux achetés } \\ \text { en dehors de la branche }\end{array} \\ \text {........ Engrais et amendements } & \text { Produits de protection des cultures }\end{array}$

ment du troupeau, grâce à l'augmentation du chargement et l'accroissement des rendements laitiers. Le passage au zéro-pâturage est par ailleurs aujourd'hui également accéléré par l'adoption du robot de traite. Ce type de système de production laitier, basé sur une ration identique tout au long de l'année à base d'ensilage de maïs et de tourteau, conduit en zéro-pâturage, équipé d'une salle de traite très performante ou d'un robot de traite, est devenu courant dans les régions de plaine en France; il est encore beaucoup plus répandu au Danemark, aux Pays-Bas, ou aux EtatsUnis, où il concerne des troupeaux de grande voire de très grande taille (plusieurs milliers de têtes aux Etats-Unis).

La logique de ce type de développement repose, comme en grandes cultures, sur un capital fixe de plus en plus conséquent par actif mais aussi sur un recours important aux consommations intermédiaires (achat d'aliments, culture du maïs, importance des fourrages stockés dans la ration au détriment du pâturage). La même logique a été privilégiée pour les autres types d'élevages.

\section{4 / Des résultats économiques contrastés : accroissement de la production en volume, mais diminution de la valeur ajoutée réelle de la branche agriculture}

La politique agricole a joué un rôle important dans les profondes transformations de l'agriculture : la garantie apportée aux prix agricoles (notamment pour les céréales, le lait ou la viande bovine), ainsi que les politiques de crédit et de restructuration foncière ont encouragé les agriculteurs et leur ont donné les moyens de réaliser des investissements et d'acquérir les nouveaux moyens techniques permettant d'accrô̂tre la productivité de leur travail. Le caractère familial des exploitations agricoles a indéniablement été déterminant : ne cherchant pas à rentabiliser au mieux leur capital, mais avant tout à rémunérer leur propre travail, les exploitants agricoles qui en avaient les moyens, bénéficiant de la sécurité des prix garantis, ont poursuivi leurs investissements à un rythme rapide depuis les années 1950.

Les Comptes de la Nation permettent d'observer les résultats de ces transfor- mations pour le secteur agricole dans son ensemble. La production agricole en volume a été multipliée par 2,2 en cinquante ans (figure 1). Les consommations intermédiaires ont augmenté en volume à un rythme à peine supérieur, permettant à la valeur ajoutée brute en volume de doubler sur la même période. L'évolution des différents postes de consommations intermédiaires apparaît très contrastée. L'utilisation des produits de protection des cultures, de l'alimentation achetée et des dépenses vétérinaires, ainsi que le recours aux services d'entreprise agricole se sont accrus rapidement jusqu'aux années 1990 et maintenus depuis au même niveau. Le volume d'engrais et amendements a diminué depuis le milieu des années 1980, mais cette évolution masque la relative stabilité de la consommation d'engrais azotés, en légère diminution seulement depuis le début des années 2000. Ces différentes consommations participent directement à l'accroissement de la productivité physique du travail. En revanche, un accroissement beaucoup moins important peut être constaté pour le poste de l'énergie, stable depuis 1975 et même une diminution des frais d'entretien des bâtiments et du matériel, évolutions à mettre en relation avec les économies d'échelle permises par l'agrandissement des structures.

L'accroissement de la production et de la valeur ajoutée brute en volume s'est accompagné d'une forte diminution de la population active. Il traduit donc la très rapide augmentation de la productivité du travail dans le secteur agricole. La productivité apparente du travail, mesurée par la valeur ajoutée brute en volume par équivalent temps plein, s'est accrue en agriculture à un rythme beaucoup plus rapide que dans les autres secteurs économiques (figure 2) depuis les années 1950. Elle a augmenté en moyenne chaque année de 6,5\% entre 1949 et 1961 (Malassis 1969) et de presque $5 \%$ entre 1969 et 1989 (Mabile 1990), contre respectivement 5,1 et 3,3\% pour l'ensemble de l'économie ; depuis 1978, elle a évolué plus de deux fois plus rapidement que dans l'ensemble de l'économie (Guihard et Lesdos 2007).

Ces gains de productivité se sont répercutés sur les prix des produits agricoles : en termes réels 3 , le prix des céréales a ainsi diminué de $75 \%$ depuis le début des années 1960, celui du lait et de la viande bovine de moitié (INSEE, séries longues IPPAP). Le différentiel sectoriel de productivité du travail se

\footnotetext{
${ }^{3}$ Evolution corrigée de l'inflation mesurée ici par l'indice de prix du Produit Intérieur Brut.
} 
Figure 2. Productivité apparente brute du travail par branche (valeur ajoutée brute en volume/actif).

$(1978=$ indice 100). Source : INSEE, Comptes de la Nation.

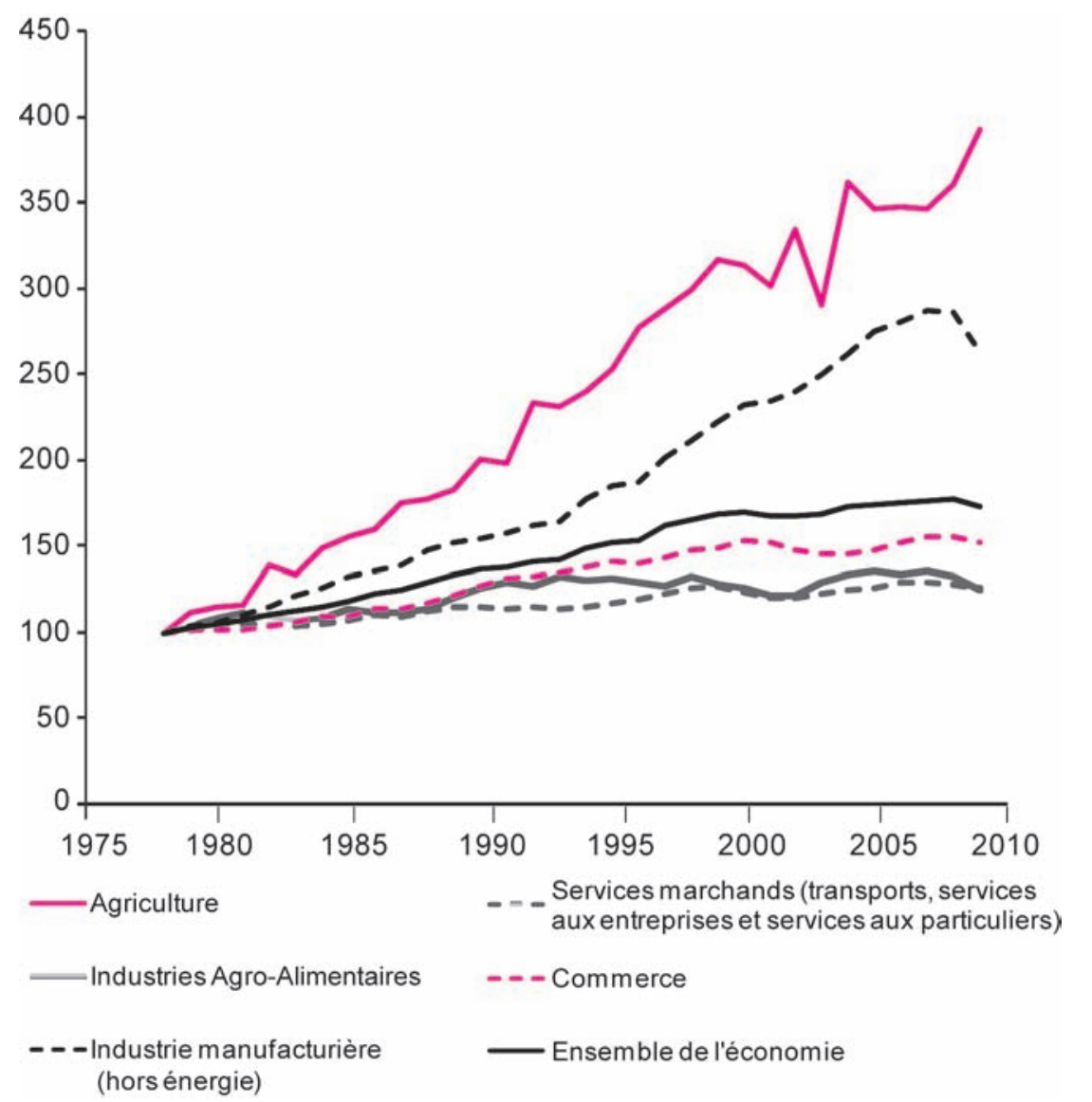

2000 et le revenu net d'entreprise agricole ne se maintient qu'avec les subventions.

Ainsi, en dépit de l'accroissement spectaculaire de la productivité physique du travail dans le secteur agricole, la valeur ajoutée et le revenu de l'agriculture en termes réels (déflatés par le prix du PIB) ne cessent de diminuer depuis le milieu des années 1970 (figure 3). Le revenu agricole par actif ne s'est globalement maintenu qu'avec la diminution du nombre d'actifs qui se poursuit à un rythme rapide : après avoir stagné de 1970 jusqu'au début des années 1990 (Desriers 2007), celui-ci a augmenté de $20 \%$ entre 1990 et 2000 , avant de diminuer ensuite pour revenir à un niveau environ $10 \%$ supérieur à celui de 1990 (INSEE, comptes provisoires de l'agriculture 2011). Cette évolution a toutes les chances de se poursuivre car les exploitations sont loin d'avoir adopté les équipements les plus performants qui existent, et ceux-ci ne cessent, avec la révolution des technologies de l'information, de la communication et de l'informatique, de repousser les limites en termes de superficie ou de nombre d'animaux par actif. traduit, en monnaie constante, par une diminution plus rapide des prix agricoles par rapport à ceux des biens, équipements et services qu'achètent les agriculteurs.

Cette évolution des prix défavorable à l'agriculture se répercute sur les résultats économiques de la branche (figure 3). Le poids des consommations intermédiaires et de la consommation annuelle de capital fixe dans la production agricole s'est considérablement accru depuis cinquante ans, de manière bien plus importante en valeur qu'en volume. Ainsi, les consommations intermédiaires sont-elles passées de $40 \%$ de la valeur de la production agricole dans les années 1960 à 50\% environ dans les trente années suivantes et à plus de $60 \%$ aujourd'hui. La part de la consommation de capital fixe dans la valeur de la production a régulièrement augmenté pour atteindre un peu plus de $15 \%$ aujourd'hui. Au total, la valeur ajoutée nette de l'agriculture ne représente plus aujourd'hui que $20 \%$ de la valeur de la production agricole contre $55 \%$ au début des années 1960. En monnaie courante, la valeur ajoutée agricole diminue depuis le début des années
Figure 3. Evolution en monnaie constante de la production, de la valeur ajoutée et du revenu de la branche agricole.

(1959 = indice 100). Source : INSEE, Comptes de la Nation.

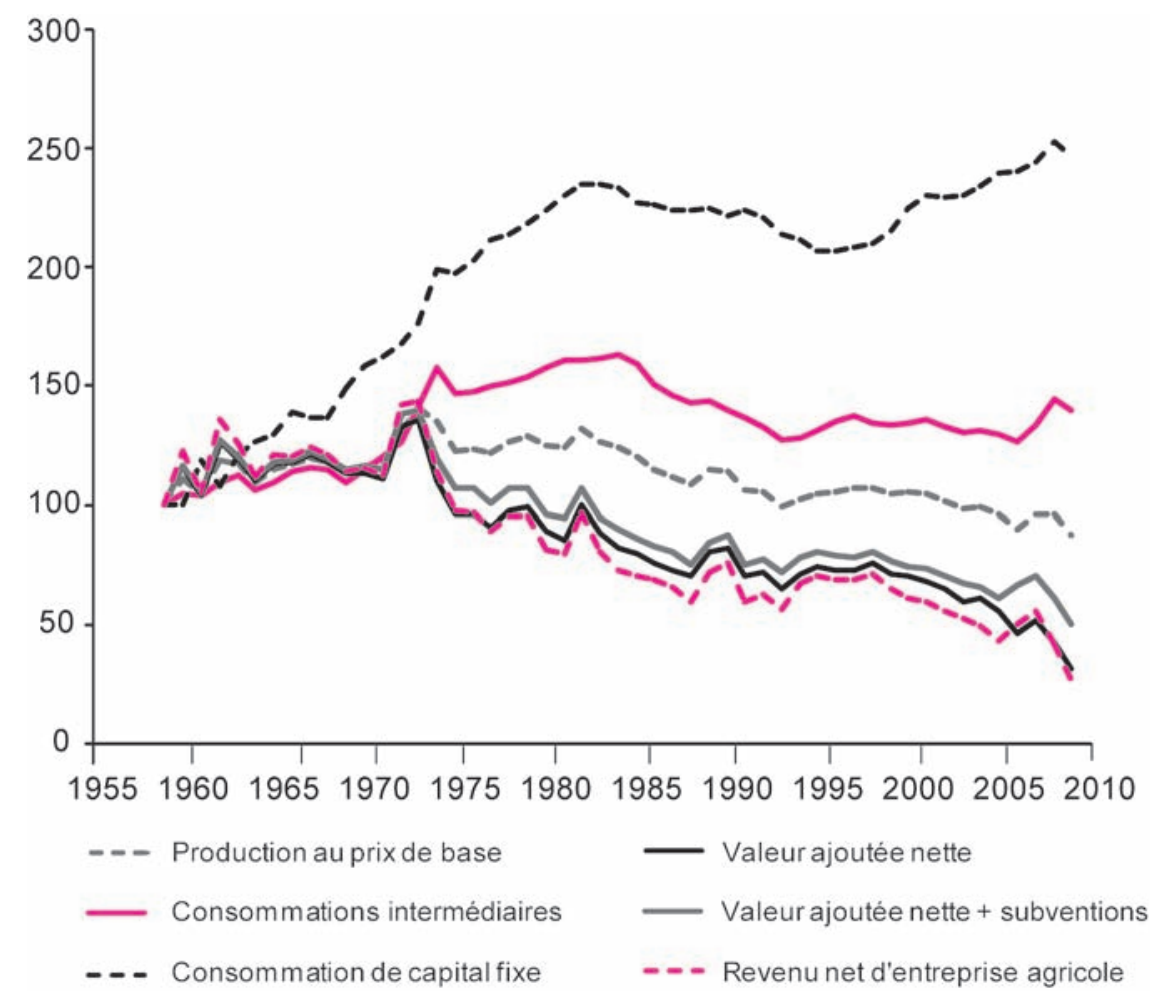




\section{3 / Productivité du travail et économie en élevage bovin allaitant : trajectoires d'évo- lution d'exploitations}

Les évolutions constatées globalement au niveau de l'agriculture française masquent des disparités entre les différentes filières et zones géographiques. Les forts gains de productivité, aussi bien en grandes cultures, cultures fourragères et production laitière, ont fortement impacté les effectifs d'herbivores. Le troupeau bovin s'est de plus recomposé : sur les 10 millions de vaches présentes en 1970, trois sur quatre étaient laitières ; en 2011, plus d'une vache sur deux est une allaitante $(7,9$ millions de vaches dont 3,7 millions de laitières et 4,2 millions d'allaitantes). Le troupeau allaitant fournit aujourd'hui près de $60 \%$ de la viande bovine produite et consommée en France.

Les bovins allaitants ont été longtemps, en France, l'apanage des montagnes du Massif Central et des plaines herbagères à faible potentialité agronomique. En 1970, le troupeau allaitant était localisé à $75 \%$ dans cinq régions : Bourgogne, Limousin, Auvergne, MidiPyrénées et Aquitaine. L'instauration des quotas laitiers a provoqué un développement du troupeau allaitant dans les zones intensives de l'Ouest. Ce troupeau s'est délocalisé, les zones herbagères et montagneuses ont ainsi vu leur part régresser de 75 à $50 \%$ de l'effectif allaitant national.

Les exploitations de ces régions d'élevage herbager classées en zone difficile sont restées spécialisées dans la production de viande bovine mais ont subi de grands bouleversements dans leurs structures et systèmes de production. Nous allons illustrer ces évolutions par les trajectoires observées d'exploitations d'élevage bovin allaitant du Bassin Charolais.

\section{1 / Productivité physique du travail et revenu en élevage bovin allaitant spécialisé}

En 20 ans, de 1990 à 2009, la superficie moyenne (SAU en ha) des exploitations d'élevage bovin allaitant en France métropolitaine (Agreste, RICA, OTEX42) a augmenté de plus de $60 \%$, le cheptel moyen de près de $65 \%$ alors que le nombre de travailleurs totaux par exploitation a baissé de 7\%. Ces résultats issus d'une «sonde» nationale de 500 à 700 exploitations (nombre variable et exploitations différentes selon l'année) représentant statistiquement l'ensemble des exploitations d'élevage

Figure 4. Evolution de la productivité du travail dans les exploitations bovines Charolais du Centre de la France.

Source : Réseau Charolais INRA, 59 exploitations.

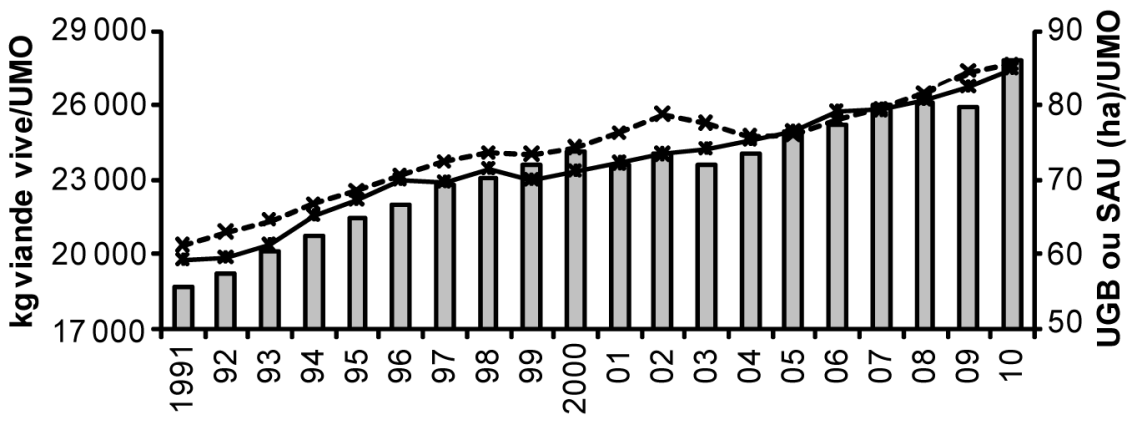

g

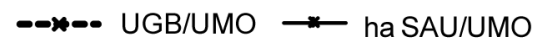

bovin allaitant, ne renseignent pas sur leurs trajectoires et leurs adaptations à ces changement structuraux.

Afin de comprendre les déterminants des évolutions des exploitations, de connaître et d'analyser les potentialités techniques et économiques des systèmes de production, une équipe de l'INRA de Clermont-Ferrand/Theix a mis en place, depuis les années 1970 un réseau d'observations sur le long terme d'exploitations d'élevage bovin allaitant Charolais du centre de la France (Lherm et al 1983). Pour l'analyse qui suit, nous allons nous appuyer sur un échantillon constant de 59 exploitations spécialisées (naisseurs stricts et naisseursengraisseurs) sur les 20 dernières années (1991-2010) de ce réseau Charolais INRA.

\section{a) Evolution des structures}

A la fin des années 1980, de nombreux GAEC père-fils se sont constitués. Au cours des années 2000, après son départ à la retraite, le père continue souvent à travailler bénévolement sur l'exploitation quelques années. Depuis le début des années 2000, le nombre de travailleurs exploitants a donc tendance à diminuer au profit des travailleurs bénévoles (parents à la retraite) qui représentent près de $5 \%$ des travailleurs totaux ces dernières années (contre moins de $1 \%$ dans les années 1990) Parallèlement, le nombre de salariés a légèrement augmenté, passant de 0,19 à 0,30 UMO entre 1991 et 2010. Les salariés et les bénévoles ne compensent pas entièrement les départs des exploitants retraités. Entre 1991 et 2010, le nombre moyen de travailleurs totaux par exploitation a baissé de $4 \%$, respectivement 2,11 et 2,03 UMO en 1991 et 2010, avec toujours une forte dominance du travail des exploitants (familiaux ou associés dans les formes sociétaires). Une incertitude pèse sur les solutions à trouver lorsque les bénévoles cesseront définitivement de travailler.
Contrairement aux grandes zones de polyculture-élevage, nous n'avons pas assisté dans le Charolais à une évolution marquante des assolements, ni à une intensification des surfaces fourragères. L'instauration, en 1992, d'un complément de prime animale pour les systèmes «extensifs», ainsi que la mise en place de la Prime au Maintien des Systèmes d'Elevage Extensifs (PMSE) (dite "prime à l'herbe») ont été suffisamment incitatives pour conforter ces systèmes. Les seuils de chargement pour l'attribution de ces aides étaient très proches des chargements observés dans la région, les surfaces en herbe et le nombre d'UGB totaux se sont donc «figés». En revanche, les tendances à l'agrandissement des structures ont été observées ici comme partout ailleurs. La SAU des exploitations a augmenté de $41 \%$ en 20 ans, et ce, de façon quasi continue à un rythme de 1,6 à $1,9 \%$ par an. La part de la Surface Fourragère Principale (SFP) dans la SAU est restée stable (autour de 80\%), l'orientation «élevage spécialisé» de ces exploitations s'est donc maintenue. La taille des troupeaux a augmenté dans les mêmes proportions que celle de la surface, soit + 36\% (179 UGB en 2010 vs 132 UGB en 1991), le chargement est donc resté stable entre 1,25 et 1,30 UGB/ha SFP.

La productivité physique du travail a par conséquent fortement augmenté (figure 4). Le nombre d'hectares de SAU, de vaches et d'UGB totaux détenus par UMO totale a augmenté respectivement de 50, 55 et $42 \%$ en 20 ans. En 2010, un travailleur produit $48 \%$ de viande vive de plus qu'en 1991 (27 $780 \mathrm{~kg}$ vifs produits/UMO en $2010 \mathrm{vs}$ $18730 \mathrm{~kg}$ en 1991).

b) Systèmes de production et résultats techniques

Les réformes successives de la PAC depuis 1992 (baisse des prix garantis compensée, en partie, par des aides directes), ainsi que les évolutions de 
Figure 5. Evolution du revenu du travail et des capitaux (RWC) par travailleur (UMO ) et par hectare de SAU dans les exploitations bovines Charolais du Centre de la France.

Source : Réseau Charolais INRA, 59 exploitations.

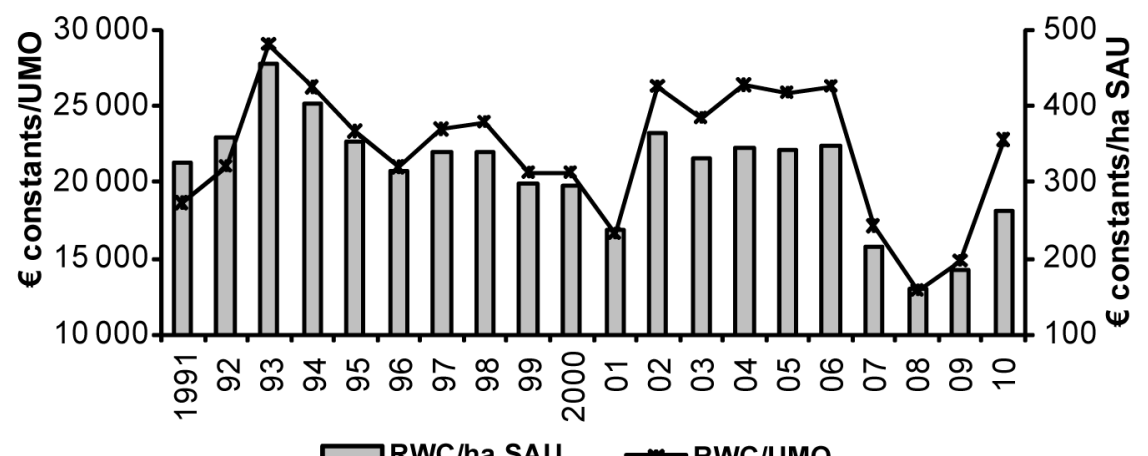

prix de marché, ont abouti à un raccourcissement du cycle de production et à une moindre diversité de types de mâles produits (Veysset et al 2005a). Deux grandes catégories représentent plus de $90 \%$ des mâles produits : les broutards (plus ou moins âgés et lourds) et les jeunes bovins gras, avec une prédominance des broutards (70\% des mâles vendus). Alors que, dans les années 1980, une même ferme pouvait produire jusqu'à 4 ou 5 types d'animaux différents $(\mathrm{du}$ jeune broutard au bœuf de 4 ans, l'interdiction des anabolisants en 1988 a été «fatale» pour la production de bœufs), la «standardisation» et la spécialisation des systèmes autour de la production de un ou deux types d'animaux ont conduit à gérer une moindre diversité d'animaux aux besoins différents dans une même ferme. Les nouveaux bâtiments d'élevage et les nouvelles techniques d'alimentation ont permis de loger et nourrir dans de bonnes conditions des lots de grande taille. La taille des troupeaux de vaches a ainsi pu augmenter sans pénaliser significativement les résultats zootechniques (Bébin et al 1995).

La forte baisse du prix des céréales depuis 1992 et donc des concentrés, a également encouragé la simplification des pratiques. La distribution de concentrés s'est faite de façon beaucoup plus libérale : $720 \mathrm{~kg} / \mathrm{UGB}$ en 2010 vs $510 \mathrm{~kg} / \mathrm{UGB}$ en 1991 (+41\% en 20 ans) alors que la productivité animale, c'està-dire la production de viande vive (kg vifs) par UGB, n'augmentait que de $5 \%$ (312 kg/UGB vs 298). Dans le même temps, le chargement et donc la disponibilité en fourrages par animal, n'ont pas évolué. La valorisation de la ressource en herbe, c'est-à-dire les $\mathrm{kg}$ de viande produits uniquement avec l'herbe, a baissé au profit des aliments concentrés faciles à acheter et de valeur alimentaire stable sur l'année. Nous observons ces dernières années une volatilité du prix des céréales avec des ble des travailleurs présents, sans distinction de statut et l'ensemble des capiCharolais du Centre de la France.

Source : Réseau Charolais INRA, 59 exploitations. taux propres de l'exploitation hors foncier. Nous appellerons ce résultat économique le revenu du travail et des capitaux (RWC).

$R W C=$ Résultats Courant + (salaires nets) - (valeur locative des terres en propriété - impôts fonciers)

Sur les vingt années étudiées, le RWC par travailleur s'est maintenu autour de $22000 € /$ UMO (en euros constants 2010 en tenant compte de l'évolution de l'indice moyen annuel des prix à la consommation INSEE), avec des variations annuelles assez conséquentes (figure 5). En 2010, les aides totales représentent $151 \%$ du RWC (hors aides, ce revenu serait négatif), la viabilité économique de ces exploitations est donc très fortement dépendante de celles-ci (Veysset et al 2005b). Les gains de 45 à $50 \%$ de productivité physique du travail ont donc juste permis de maintenir les revenus.

Le RWC/ha de SAU présente également de grandes variations annuelles, mais, globalement, la tendance à la baisse est significative $\left(-8,06 € / \mathrm{ha} / \mathrm{an}, \mathrm{r}^{2}=\right.$ $0,44)$ du fait, notamment, de la hausse des charges fixes (figure 6). La baisse du prix de la viande de $26 \%$ en 20 ans ayant été compensée par l'augmentation des aides totales et par le léger accroissement de la productivité animale, le produit par ha est resté stable. Les charges proportionnelles par ha ont également été relativement stables sur la période étudiée, la forte augmentation des quantités de concentrés distribués a été compensée par la baisse conséquente de leur prix unitaire. L'augmentation du prix unitaire des engrais et des produits phytosanitaires a entraîné une diminution de leur utilisation. Alors qu'avec l'augmentation de la taille des exploitations nous pouvions nous attendre à une diminution des frais de mécanisation et de bâtiment (notion d'économie d'échelle), ceux-ci ont augmenté respectivement de 30 et $20 \%$. Les autres charges fixes ont peu évolué. Parmi les charges de mécanisation à l'hectare, les frais de carburants et les amortisse-

Figure 6. Evolution des charges fixes par ha de SAU dans les exploitations bovines

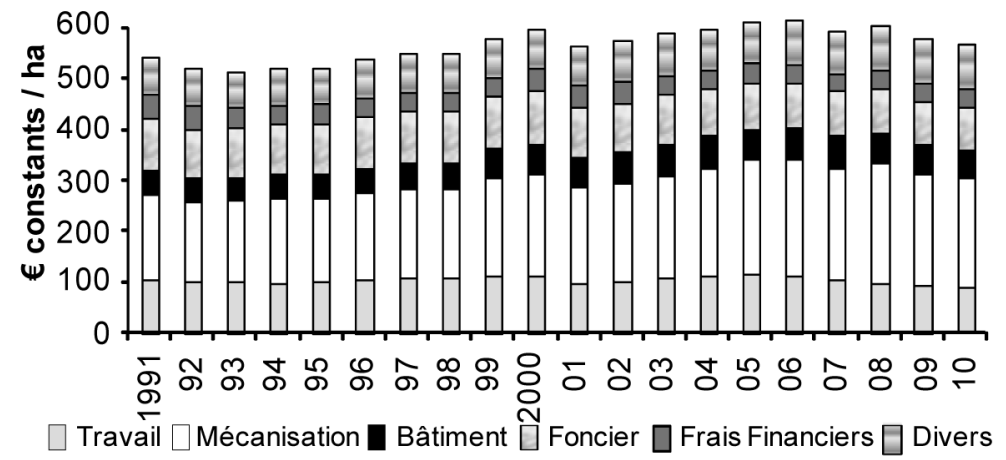


Figure 7. Evolution du capital par ha et par UMO dans les exploitations bovines Charolais du Centre de la France.

Source : Réseau Charolais INRA, 59 exploitations.
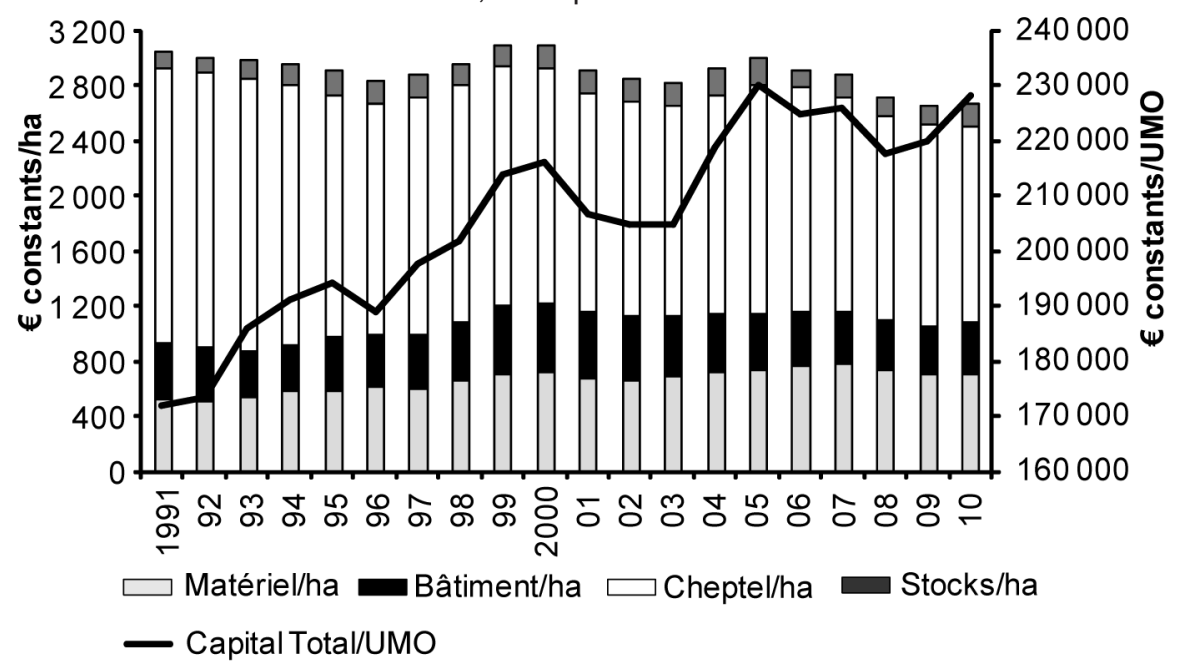

ments (consommation annuelle du capital matériel, amortissements dégressifs de 10 à $15 \%$ par an selon le matériel) ont augmenté respectivement de 61 et $18 \%$, les frais d'entretien sont restés stables; de même, pour les bâtiments, ce sont les charges d'amortissement (amortissements linéaires sur 10 à 20 ans) qui ont augmenté.

Contrairement aux observations réalisées sur l'ensemble de l'agriculture française (figure 1), il n'y a pas eu effet de dilution de la consommation d'énergie, des charges de structure et notamment de l'utilisation du matériel et des bâtiments avec l'augmentation de la taille des exploitations. La notion d'économie d'échelle, qui a guidé l'évolution de bon nombre de structures productives agricoles, n'est donc pas valide dans ces exploitations d'élevage bovin allaitant.

Ces producteurs de viande bovine Charolaise n'ont donc pas retenu leurs gains de productivité, mais les ont «distribués» (Butault et al 1995) presque intégralement, sous forme de baisse de prix et de consommation de matériel.

Ces résultats sont corroborés par l'étude de l'évolution des coûts de production du kilogramme de viande vive produit, incluant la rémunération de la main-d'œuvre exploitante et des capitaux propres (Institut de l'Elevage 2010a). Ce coût de production est quasi stable, 3,29 €/kg vif, sur 20 ans. Seul le poste charges fixes est en forte progression : +20 centimes d' $€ / \mathrm{kg}$ soit $+18 \%$, alors que le poste rémunération de la main-d'œuvre exploitante est en baisse de $28 \%$ (il faut moins de travail pour produire un $\mathrm{kg}$ de viande). Sur les cinq dernières années, les charges fixes hors main-d'œuvre représentent $42 \%$ du coût de production et les charges de travail totales en représentent $27 \%$.

\section{2 / Contribution du capital à} l'accroissement de la productivité poste en valeur de capital total d'une exploitation bovine allaitante. Si le capital total détenu par ha de SAU des exploitations en élevage bovin allaitant Charolais a baissé de $13 \%$ en 20 ans (figure 7), ce n'est pas grâce à une meilleure utilisation du capital sur de plus grandes structures, mais uniquement grâce à la baisse de la valeur, quasiment équivalente, du cheptel sur la même période.

L'accroissement de la productivité du travail a pu se réaliser grâce à la modernisation des exploitations (accompagnant la spécialisation des troupeaux et la simplification des pratiques) principalement par des investissements lourds et continus en matériel. Ces investissements matériels ne sont cependant pas tous réalisés dans un but productif en vue d'améliorer le revenu (ils participent à la baisse du revenu par les amortissements et les frais financiers générés), mais pour certains, dans un intérêt fiscal. Dans le cadre de projets d'installation d'un fils (GAEC père-fils) avec obtention de nouveaux droits (gratuits) de PMTVA, de nouvelles stabulations pour loger l'ensemble des animaux et faciliter le travail, se sont construites. Par ailleurs, beaucoup d'éleveurs concernés par la mise aux normes des bâtiments et installations existants ont préféré investir dans un nouveau bâtiment en utilisant les subventions qui auraient été nécessaires pour moderniser l'ancien. On a ainsi assisté à un fort renouvellement avec modernisation du parc de bâtiments. Ces investissements dans les bâtiments sont relativement lourds en valeur absolue, mais, contrairement au matériel, sont réalisés une
Le cheptel représente le premier seule fois, avec une durée d'amortissement longue (20 ans).

Au final, malgré l'agrandissement des fermes, le capital matériel détenu par ha de SAU a progressé de $36 \%$ en 20 ans. Le capital bâtiment est resté stable ; et en 2010, il est deux fois moins important par ha que celui en matériel. Le cheptel qui représentait $65 \%$ du capital total d'une exploitation Charolaise en 1991 n'en représente que 53\% en 2010 , alors que le capital matériel voit sa part passer de 17 à $27 \%$.

Malgré la baisse du capital par ha, le nombre d'ha détenus par travailleur (tous types de travailleurs confondus) ne cessant d'augmenter, le capital détenu par travailleur s'accroît de $33 \%$ en 20 ans pour atteindre près de $230000 € /$ UMO en 2010. En 2010, un travailleur en exploitation d'élevage bovin allaitant doit engager $20 \%$ de capital de plus qu'en 1991 (en euros constants) pour une espérance de revenu identique. Ceci pénalise évidemment l'entrée des jeunes dans cette profession et explique pourquoi les exploitants sortants ne trouvent pas toujours facilement un acquéreur unique pour leur outil de production (Veysset et al 2008).

\section{4 / Relation entre producti- vité physique du travail et indicateurs économiques en élevage d'herbivores}

Ces observations sur les tendances passées donnent lieu à penser qu'il existe une corrélation, plus ou moins significative selon les productions, entre productivité physique du travail et revenu. Pour les filières d'herbivores, la productivité est également liée à la zone et au système de production. Au-delà d'une analyse transversale de ces relations, nous décrirons pour chaque filière les principaux repères de productivité physique du travail, le lien avec les performances économiques et le besoin en capital qui, comme cela a été souligné précédemment, est très lié à l'accroissement de la productivité du travail et devient un point déterminant pour la transmissibilité du système.

L'analyse statistique porte sur les données de l'année 2009. Cependant, pour prendre en compte le caractère exceptionnel de la conjoncture laitière bovine de cette année-là (baisse de revenu de près de $50 \%$ ), nous avons également analysé les données de l'année 2008.

\section{1 / L'échantillon}

L'analyse repose sur les résultats des 1441 exploitations du dispositif des 
Réseaux d'Elevage 4 . Elle a été rendue possible grâce à la disponibilité conjointe de données techniques et économiques. Cet échantillon est composé d'exploitations de taille moyenne à grande (les petites structures sont peu représentées dans le dispositif mobilisé).

Les exploitations combinant un élevage herbivore avec une production horssol, des cultures pérennes ou spéciales ou encore de la transformation fromagère, n'ont pas été retenues du fait d'effectifs insuffisants. A contrario, toutes les exploitations comprenant des grandes cultures $(43 \%$ des exploitations du dispositif au sens des OTEX - polyculture élevage voire de grandes cultures avec de l'élevage) ont été conservées. De grandes structures sociétaires diversifiées présentant des volumes de production importants et une productivité du travail souvent élevée ont également été intégrées dans l'échantillon étudié.

Toutes les exploitations ont été typées selon les conventions de la statistique agricole qui déterminent l'orientation technico-économique (OTEX) et la classe de dimension (CDEX). Nous avons finalement retenu trois grandes familles d'OTEX : $i$ ) les systèmes spécialisés herbivores avec une ou plusieurs productions animales «HER» (OTEX 41-Bovins lait, 42-Bovins viande, 43-Bovins mixtes, 44-Autres herbivores et 71-Polyélevage - Herbivores) ; ii) les systèmes de polyculture-élevage «POLY» (OTEX 60-Polyculture et 81Grandes cultures et herbivores); iii) les exploitations céréalières avec la présence d'élevage «GCU» (OTEX 13Céréales - Oléagineux - Protéagineux et 14-Autres grandes cultures).

Parmi les trois filières laitières, ce sont les systèmes ovins qui sont les plus spécialisés en élevage alors que, pour les bovins et caprins, les systèmes de polyculture représentent respectivement 20 et $30 \%$ des exploitations (les associations avec une part importante de grandes cultures -GCU- étant cependant peu fréquentes). Pour les filières viandes bovine et ovine, un peu plus de $10 \%$ des exploitations se caractérisent par une prédominance de grandes cultures dans le système.
Le nombre total d'UMO par exploitation varie de 0,5 à 6 . La majorité des exploitations (53\%) n'a pas de salarié, ni de bénévole. La main-d'œuvre salariée représente $9 \%$ du total des UMO de l'échantillon et son importance va de pair avec la dimension des exploitations. Elle est plus fréquente dans les systèmes caprins et au sein des systèmes associant une production bovine (lait ou viande) avec des céréales. La part de bénévolat est plus faible (6\%). Les systèmes ovins laitiers en comptent le plus $(12 \%)$ et les systèmes laitiers bovins et caprins sont ceux qui y ont le moins recours $(<5 \%)$. La part de cette maind'œuvre est inversement proportionnelle à celle de la main-d'œuvre rémunérée (14\% de la main-d'œuvre totale dans les plus petites exploitations contre $3 \%$ pour les plus grandes).

$\mathrm{Au}$ niveau de la main-d'œuvre rémunérée, 3 classes ressortent : un tiers des exploitations a moins de 1,4 UMO, $40 \%$ comptent 1,4 à 2 UMO et un quart compte plus de 2 UMO. Les exploitations allaitantes ovines et bovines sont pour près de la moitié d'entre elles dans la première catégorie alors que celles de la filière bovine laitière et les associations avec des grandes cultures prédominent dans la troisième.

Pour la suite des traitements, nous ne prendrons en compte que la main-d'œuvre rémunérée (UMOr), salariée ou non.

\section{2 / Productivité physique du travail et indicateurs écono- miques selon les filières ani- males}

\section{a) Analyse transversale des filières}

A partir d'une affectation de la maind'œuvre aux différents ateliers des exploitations (méthode décrite dans la partie 1) et d'un regroupement par filière animale principale, nous avons croisé la productivité physique et les principaux critères économiques de l'atelier (tableau 1).

Plusieurs tendances se dessinent (en lien avec les évolutions observées pour la branche agricole) :

- Le produit brut total est logiquement très fortement corrélé à la productivité physique du travail pour toutes les filiè- res. La relation avec les aides est nettement moins forte et devient même négative en filière caprine (historiquement moins concernée).

- L'augmentation de la productivité du travail repose sur une part croissante d'intrants et de capital dans toutes les filières. L'impact des intrants sur 1'efficience économique, appréhendée par le critère $\mathrm{EBE} / \mathrm{PB}$, est systématiquement négatif. L'accroissement des amortissements lié à celui du capital dégrade également très fortement la relation entre productivité du travail et valeur ajoutée nette pour les conjonctures les moins favorables (cas en 2009 pour les filières bovines). Il en va de même pour le revenu courant.

Il est clair que le résultat de ce type d'analyse est très dépendant de la conjoncture économique de l'année. Nous aurons l'occasion de l'illustrer en production de lait de vache à travers deux années ayant connu des conjonctures très contrastées, 2008 et 2009.

Le lien entre productivité physique et revenu a été analysé pour les différents systèmes au sein des filières à l'aide de régressions univariées.

\section{b) Les productions laitières bovines,} ovines et caprines

En production laitière, le volume de lait commercialisé a été retenu comme unité pour quantifier la productivité physique des exploitations. C'est un indicateur facile à déterminer et très utilisé. Il ne tient pas compte du coproduit viande (animaux de réforme et animaux jeunes - veaux, agneaux et chevreaux) qui ne représente qu'une part marginale de travail physique.

Les volumes de lait commercialisé par unité de main-d'œuvre rémunérée sont généralement différents entre les systèmes de plaine et ceux de montagne. Le handicap dû au milieu impacte significativement la productivité du travail : les systèmes d'élevage de piémont et de montagne enregistrent une productivité physique du travail inférieure à celle des systèmes de plaine (figure 8).

Pour les zones de montagne, nous avons également distingué les exploita-

\footnotetext{
${ }^{4}$ L'approche globale (structurelle, technique et économique) des exploitations d'élevage mise en œuvre en France dans les «Réseaux d’Elevage pour le Conseil et la Prospective (RECP)» depuis le début des années 1980 (Lebrun 1983) repose sur un partenariat associant des éleveurs volontaires, les Chambres d'Agriculture et l'Institut de l'Elevage. Ce dispositif s'appuie aujourd'hui sur un échantillon raisonné de 1420 exploitations des filières bovines, ovines et caprines et, pour certaines régions, d'exploitations supplémentaires suivies selon la même méthodologie. Le suivi approfondi et le traitement des données recueillies, combinés à l'expertise des acteurs de ce dispositif permettent de mettre au point, de modéliser et de décrire des systèmes de production efficients et cohérents, à des niveaux d'optimisation compatibles avec leurs contextes socio-économiques et pédoclimatiques (Delaveau et al 1999). Les Réseaux d'élevage contribuent ainsi à l'élaboration de références globales et/ou thématiques utilisées pour le conseil par les agents de développement et pour l'aide à la décision par les professionnels et les acteurs publics. Les critères de choix des exploitations (structures, performances et modes de conduite) et le suivi rapproché sur plusieurs années répondent à un objectif de connaissance de la diversité des systèmes plus que de représentativité.
} 
Tableau 1. Coefficients de corrélations entre la productivité physique du travail dans les filières herbivores (en unité de produit par UMOr) et différents indicateurs économiques.

Source : Réseaux d'elevage 2009.

\begin{tabular}{|l|c|c|c|c|c|}
\hline & $\begin{array}{c}\text { Bovin Lait } \\
\text { Lait produit } \\
\text { (L/UMOr) }\end{array}$ & $\begin{array}{c}\text { Caprin } \\
\text { Lait produit } \\
\text { (L/UMOr) }\end{array}$ & $\begin{array}{c}\text { Ovin lait } \\
\text { Lait produit } \\
\text { (L/UMOr) }\end{array}$ & $\begin{array}{c}\text { Bovin Viande } \\
\text { Viande vive } \\
\text { (kg/UMOr) }\end{array}$ & $\begin{array}{c}\text { Ovin Viande } \\
\text { Equivalent carcasse } \\
\text { d'agneaux kg/UMOr }\end{array}$ \\
\hline Nombre d'exploitations & $\mathrm{n}=530$ & $\mathrm{n}=75$ & $\mathrm{n}=50$ & $\mathrm{n}=491$ & $\mathrm{n}=295$ \\
\hline $\begin{array}{l}\text { Résultats économiques } \\
\text { de l'atelier }\end{array}$ & & & & \\
\hline Produit Brut (PB) en $€ /$ UMOr & 0,93 & 0,97 & 0,94 & 0,89 & 0,90 \\
\hline Produit brut hors aides en $€ /$ /UMOr & 0,94 & 0,98 & 0,96 & 0,92 & 0,95 \\
\hline Aides en €/UMOr & 0,42 & $-0,16$ & 0,50 & 0,48 & 0,35 \\
\hline Intrants en $€ /$ /UOr & 0,86 & 0,92 & 0,70 & 0,90 & 0,73 \\
\hline Valeur ajoutée brute en $€ /$ /UMOr & 0,48 & 0,79 & 0,73 & 0,27 & 0,59 \\
\hline Valeur ajoutée nette $€ / U M O r$ & 0,15 & 0,60 & 0,36 & $-0,09$ & 0,37 \\
\hline $\begin{array}{l}\text { Excédent Brut d'Exploitation (EBE) } \\
\text { en } € / U M O r\end{array}$ & 0,53 & 0,79 & 0,82 & 0,41 & 0,63 \\
\hline EBE/PB en\% $\%$ & $-0,32$ & $-0,10$ & $-0,04$ & $-0,34$ & $-0,10$ \\
\hline $\begin{array}{l}\text { Résultat courant avant impôts } \\
\text { en } € / U M O r\end{array}$ & 0,14 & 0,53 & 0,50 & $-0,01$ & 0,43 \\
\hline $\begin{array}{l}\text { Capital d'exploitation hors foncier } \\
\text { en } € / U M O r\end{array}$ & 0,64 & 0,55 & 0,69 & 0,78 & 0,47 \\
\hline
\end{tabular}

tions en agriculture biologique et celles avec une production laitière sous signe de qualité (AOP des montagnes de l'Est). En plaine, la part de grandes cultures a été prise en compte. Enfin, les exploitations équipées d'un robot de traite ont également fait l'objet d'une catégorie spécifique.

Pour les systèmes conventionnels de plaine, nous observons un gradient croissant de productivité physique du travail pour les exploitations des grandes régions d'élevage, celles de polyculture élevage situées en région de grandes cultures (où les quotas disponibles sont importants suite à la spécialisation croissante des exploitations vers les grandes cultures) et celles équipées d'un robot de traite (pour lesquelles la mise en place de cet équipement permet

Figure 8. Productivité physique du travail des systèmes bovins laitiers.

Source : Réseaux d'élevage 2009.

La variabilité de la productivité physique de la main-d'oeuvre est présentée graphiquement sous forme de box-plot (1er décile, $1^{\text {er }}$ quartile, médiane, 3ème quartile et gème décile).

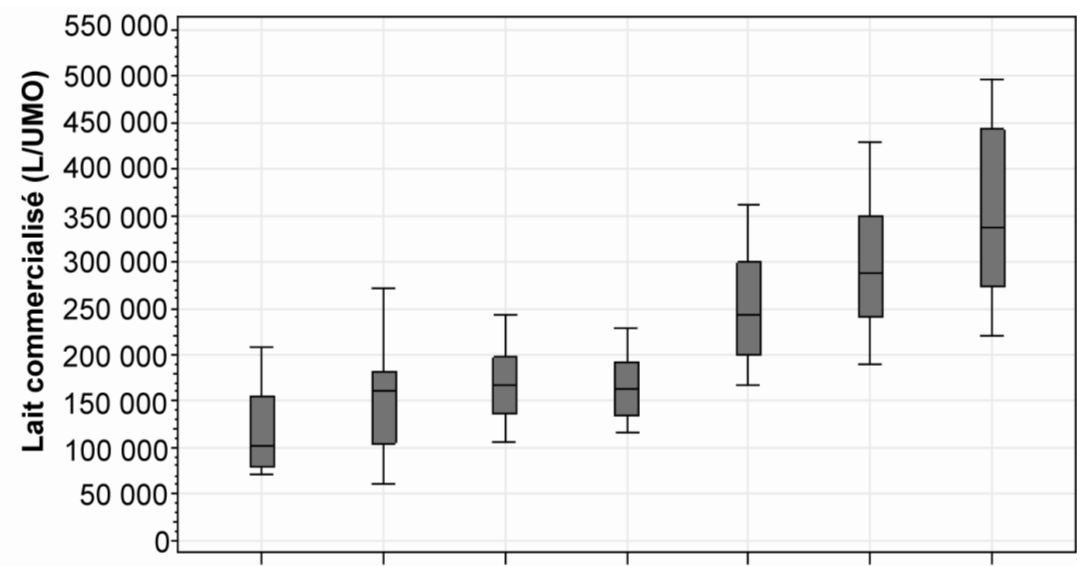

Bovins lait

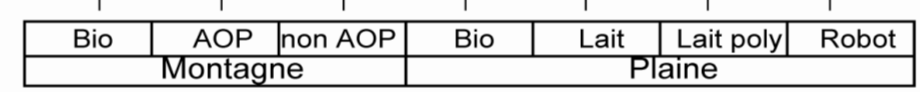

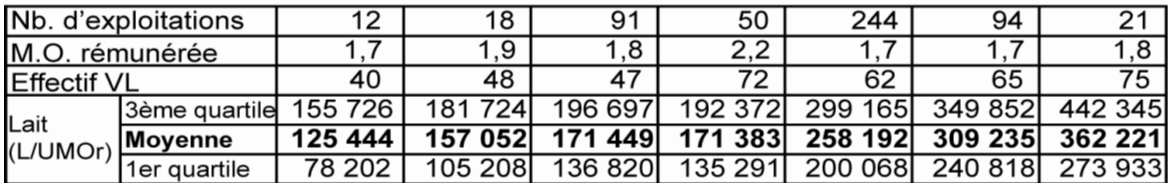

de franchir un nouveau cap de productivité physique du travail). L'écart entre les deux extrêmes atteint 100000 litres par unité de main-d'œuvre.

En montagne, les systèmes en agriculture biologique ont la plus faible productivité avec 120000 litres en moyenne par unité de main-d'œuvre, les mêmes systèmes en plaine produisant 50000 litres de plus (figure 8).

En 2009, le revenu est très peu corrélé avec la productivité $(\mathrm{r}=0,14)$. Dans ce type de conjoncture exceptionnelle (baisse du prix du lait standard de $20 \%$ par rapport à 2008 et prix des intrants élevés - revenu divisé par deux), les systèmes positionnés sur une démarcation de produit (laits issus de l'agriculture biologique ou autres AOP des montagnes de l'Est) obtiennent un revenu par unité de main-d'œuvre plus important, comme le montre par exemple la comparaison entre les résultats des exploitations laitières en AOP Comté (32 k€ RCAI/UMOr pour une productivité de 180000 L/UMOr) et ceux des exploitations de l'Ouest en système maïs dominant $(25 \mathrm{k} €$ pour une productivité de 250000 litres) (Institut de l'Elevage 2011b).

A contrario, avec une conjoncture favorable (prix du lait standard élevé) comme celle de 2008, le revenu est plus fortement corrélé avec la productivité $(r=0,43)$, et cette conjoncture convient beaucoup mieux aux systèmes les plus productifs : par exemple $37 \mathrm{k} € \mathrm{RCAI} /$ UMOr avec une productivité de 240000 L/UMOr pour le même système de l'Ouest contre $26 \mathrm{k} € / \mathrm{UMOr}$ pour une 
Tableau 2. Résultats des modèles de régressions expliquant le revenu courant avant impôts par la productivité du travail et l'efficience économique en élevage laitier.

Source : Réseaux d'élevage.

Régressions établies sur l'échantillon complet des systèmes lait «conventionnel éleveurs et polyculteurs» en plaine pour les années 2008 et 2009.

\begin{tabular}{|l|l|c|c|c|c|c|c|}
\hline \multicolumn{2}{|c|}{} & \multicolumn{3}{|c|}{2008} & \multicolumn{3}{c|}{2009} \\
\cline { 3 - 8 } \multicolumn{2}{|c|}{} & $\mathrm{n}$ & $\begin{array}{c}\text { Coefficient } \\
\text { standardisé }\end{array}$ & p-value & $\mathrm{n}$ & $\begin{array}{c}\text { Coefficient } \\
\text { standardisé }\end{array}$ & p-value \\
\hline Eleveurs & Productivité & 180 & 0,52 & 0,0001 & 256 & 0,42 & 0,0001 \\
\hline & Efficience économique & & 0,70 & 0,0001 & & 0,77 & 0,0001 \\
\hline Polyculteurs & Productivité & 80 & 0,35 & 0,0001 & 93 & 0,30 & 0,0001 \\
\hline & Efficience économique & & 0,76 & 0,0001 & & 0,73 & 0,0001 \\
\hline
\end{tabular}

productivité de 156000 litres en lait à Comté (Institut de l'Elevage 2010b). Une telle sensibilité à la conjoncture permet de mettre en relief l'importance de la cohérence et de l'efficience des systèmes dans la détermination du résultat économique.

Cela nous a incité à réaliser un traitement complémentaire des données 2008 et 2009 pour les deux types conventionnels de plaine en introduisant le ratio Excédent Brut d'Exploitation/Produit Brut. Les résultats (tableau 2) sont exprimés en coefficients standardisés $\left(\beta_{\text {standardisé }}=\beta^{*} \sigma_{x} / \sigma_{y}\right)$, ce qui permet de comparer les différents coefficients entre eux, l'unité commune étant l'écart-type.

Pour les deux types et les deux années, l'augmentation du RCAI/UMOr induite par l'efficience économique est nettement supérieure à celle liée à l'augmentation de la productivité. L'écart entre productivité et efficience économique est plus important en 2009 pour les systèmes «éleveurs» et quasiment constant entre les deux années pour les systèmes «polyculteurs». Les systèmes les plus consommateurs d'intrants se sont trouvés très pénalisés et ont dû recourir, pour les moins solides financièrement, à des emprunts de trésorerie.

En production de lait de chèvre, la comparaison se limite aux systèmes de plaine et de montagne (les systèmes fromagers caprins qui représentent près de la moitié des exploitations laitières caprines en 2009 (Source SSP Cheptel) ne sont pas retenus dans cette étude (cf. § 4.1)). La productivité physique moyenne du travail des systèmes caprins de plaine est de 150000 litres/ UMOr, soit $50 \%$ supérieure à celle de montagne. Dans les deux situations, la variabilité est forte (tableau 3 ) avec des exploitations très productives dans les deux zones (et un montant d'aides le plus faible de toutes les filières étudiées). Le lien entre la productivité et le RCAI est nettement plus fort que pour le lait de vache $(r=0,53)$, ceci étant confir- mé par les résultats du modèle de régression appliqué sur les exploitations de plaine. La conjoncture 2009 a été favorable aux revenus des élevages caprins (fourrages de qualité, prix des aliments à la baisse et poursuite de la hausse du prix du lait). Avec la crise que traverse la filière depuis 2010 (prix du lait à la baisse), les éleveurs caprins pourraient se retrouver dans la même situation que les éleveurs bovins laitiers en 2009.

En production de lait de brebis, la productivité moyenne est proche de $50000 \mathrm{~L} / \mathrm{UMOr}$. L'écart entre la productivité du Rayon de Roquefort et celle des Pyrénées n'est pas très important, même si les exploitations de la zone Roquefort sont de plus grandes dimensions. La liaison du RCAI avec la productivité est aussi significative $(\mathrm{r}=0,50)$.

Pour ces deux filières, les conjonctures 2008 et 2009 ont été beaucoup plus stables et donc, pour cette dernière année, plus favorable que pour les bovins laitiers.

\section{c) Les productions de viande bovine} et ovine

La grande diversité des systèmes de production en viande bovine ne permet pas une comparaison aussi facile et

Tableau 3. Productivité physique du travail des systèmes ovins et caprins laitiers. Source : Réseaux d'élevage 2009.

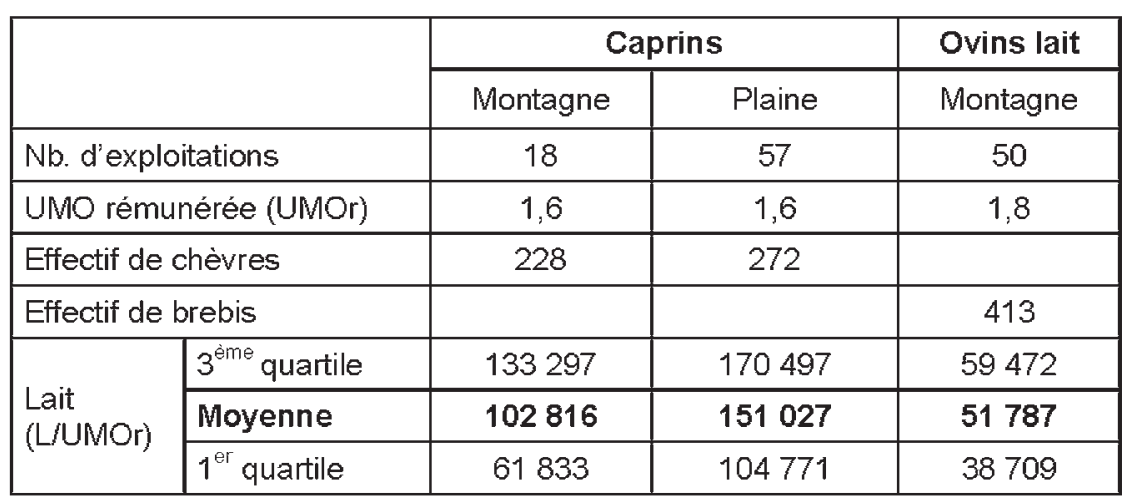

directe que pour la production laitière. Par exemple, la productivité d'un système naisseur repose sur une part importante de cheptel de souche (à faible croissance) dans le troupeau à la différence d'un système spécialisé dans l'engraissement de jeunes bovins ayant recours à des achats de broutards (à forte croissance).

Parmi les systèmes naisseurs (tableau 4), ce sont les exploitations en agriculture biologique qui observent la plus faible productivité de la maind'œuvre avec 18000 kilos de viande vive/UMOr. Les exploitations conventionnelles avec un niveau de chargement inférieur ou égal à 1,2 UGB/ha de SFP, principalement localisées dans le grand bassin allaitant, sont nettement plus productives (plus de $45 \%$ par rapport à leurs homologues en agriculture biologique). Les systèmes les plus intensifs au niveau de la conduite fourragère $(>1,2 \mathrm{UGB} / \mathrm{ha})$ apparaissent les plus productifs.

En ce qui concerne les systèmes naisseurs-engraisseurs, les producteurs de veaux sous la mère, dont les pratiques d'élevage sont «consommatrices» de main-d'œuvre (tétée contrôlée des veaux), ont logiquement la plus faible productivité (autour de $15000 \mathrm{~kg}$ / UMOr) alors que le système avec des 
Tableau 4. Productivité physique du travail des élevages de bovins à viande selon le type de système. Source : Réseaux d'élevage 2009.

\begin{tabular}{|c|c|c|c|c|c|c|c|c|}
\hline & \multicolumn{3}{|c|}{ Naisseurs } & \multicolumn{3}{|c|}{ Naisseurs-eng raisseurs } & \multirow[b]{2}{*}{$\begin{array}{c}\text { Engraisseurs } \\
\text { Jeunes } \\
\text { bovins }\end{array}$} \\
\hline & & Bio & $\begin{array}{c}\leq 1,2 \\
\text { UGB/ha } \\
\text { SFP }\end{array}$ & $\begin{array}{c}>1,2 \\
\text { UGB/ha } \\
\text { SFP }\end{array}$ & Veaux & $\begin{array}{l}\text { Jeunes } \\
\text { bovins }\end{array}$ & Bœufs & \\
\hline \multicolumn{2}{|c|}{ Nb. d'exploitations } & 30 & 119 & 140 & 14 & 135 & 15 & 17 \\
\hline \multicolumn{2}{|c|}{ UMO rémunérée (UMOr) } & 1,4 & 1,6 & 1,3 & 1,4 & 1,5 & 1,1 & 0,7 \\
\hline \multicolumn{2}{|c|}{ Effectif de vaches allaitantes } & 62 & 85 & 77 & 64 & 86 & 60 & 116 UGB \\
\hline \multirow{3}{*}{$\begin{array}{l}\text { Production } \\
\text { brute de viande } \\
\text { vive (kg/UMOr) }\end{array}$} & $3^{\text {éme }}$ quartile & 22272 & 32730 & 37632 & 18819 & 45513 & 42684 & 133717 \\
\hline & Moyenne & 18195 & 26152 & 30759 & 14480 & 38780 & 30877 & 116431 \\
\hline & $1^{\text {er }}$ quartile & 12608 & 17511 & 21101 & 8156 & 28677 & 20451 & 88033 \\
\hline
\end{tabular}

jeunes bovins produit en moyenne 39000 kilos de viande/UMOr, soit la plus forte productivité de tous les systèmes allaitants. Quant aux engraisseurs, ils atteignent des niveaux moyens plus importants, avec 115000 kilos de viande vive/UMOr.

Sur l'ensemble des exploitations, le lien entre productivité de la maind'œuvre et produit brut est là aussi très important $(\mathrm{r}=0,89)$ mais devient inexistant avec le RCAI. Une analyse selon les systèmes de production montre une relation faible mais positive pour les systèmes naisseurs. La conjoncture 2009, marquée par de très faibles revenus pour ces systèmes, a probablement un impact sur ces résultats. En 2008, comme en 2009, la relation entre valeur ajoutée nette et productivité physique du travail est quasi-nulle (respectivement 0,04 et - 0,09), ce qui rend l'acte de production peu rémunérateur ces années-là. La filière bovine à viande se trouve devant le défi d'améliorer son efficience économique car, en plus de la dépendance aux intrants, comme pour les autres filières, elle se caractérise par la plus forte corrélation entre le capital et la productivité physique $(\mathrm{r}=0,78)$.

En production ovine allaitante, l'évaluation de la productivité est basée sur les kilos de carcasse «agneaux». Cela se justifie d'un point de vue économique car, comme en viande porcine et contrairement à la viande bovine, les réformes sont beaucoup moins bien valorisées que les produits «jeunes», avec un prix par $\mathrm{kg}$ inférieur d'environ $50 \%$.

Nous avons distingué 4 grands types de systèmes de production : les systèmes «fourragers» avec un chargement supérieur à 1,4 UGB/ha, les systèmes «herbagers» $(<1,4 \mathrm{UGB} / \mathrm{ha})$ et deux types de systèmes «pastoraux» («pastoraux mineurs» et «pastoraux majeurs» selon l'importance du recours aux surfaces pastorales). La productivité physique du travail du système «fourrager»" est supérieure à $11000 \mathrm{~kg}$ d'équivalents carcasses d'agneaux/UMOr (tableau 5). Celle des «herbagers» est inférieure de $25 \%$. Ce sont les systèmes «pastoraux»" qui ont la plus faible productivité, et ce d'autant plus que la part du pastoralisme s'accroît : la taille de cheptel supérieure en comparaison des autres types de systèmes ne compense pas la moindre productivité numérique à la brebis et le moindre poids des agneaux.

Comme pour toutes les autres filières, le lien entre la productivité physique du travail et le produit brut est très net $(\mathrm{r}=0,90)$. La relation globale entre la productivité et les aides en ovins allaitants est une des plus faibles $(r=0,35)$, ce qui s'explique probablement par le fait qu'une partie des aides du second pilier ne sont pas directement proportionnelles au volume de production, donc à la productivité physique du travail. La corrélation globale entre la productivité et le revenu est de 0,43 .

d) Capital d'exploitation et productivité du travail

Comme nous l'avons mentionné dans l'analyse transversale, la relation entre le montant du capital d'exploitation hors achat de foncier (matériel, bâtiments améliorations foncières, cheptel) et la productivité physique du travail est très significative dans toutes les filières.

En valeur absolue par UMO, le montant varie de 1 à plus de 2 selon les filières (tableau 6). Au sein des filières laitières, ce sont les exploitations laitières bovines et ovines qui engagent le plus de capitaux, alors qu'en production de lait de chèvres, le capital est le moins important. En production de viande, le contraste est très fort entre les deux espèces. Les exploitations allaitantes bovines sont celles qui engagent le plus de capitaux et la répercussion financière en impacte négativement les perfor-

Tableau 5. Productivité physique du travail des élevages ovins allaitants selon le type de système.

Source : Réseaux d'élevage 2009.

\begin{tabular}{|c|c|c|c|c|c|}
\hline & & Fourragers & Herbagers & $\begin{array}{l}\text { Pastoraux } \\
\text { mineurs }\end{array}$ & $\begin{array}{c}\text { Pastoraux } \\
\text { majeurs }\end{array}$ \\
\hline \multicolumn{2}{|l|}{ Nb. d'exploitations } & 82 & 143 & 54 & 16 \\
\hline \multicolumn{2}{|l|}{ M.O. rémunérée } & 0,9 & 1,1 & 1,5 & 1,4 \\
\hline \multicolumn{2}{|l|}{ Effectif de brebis } & 454 & 490 & 533 & 543 \\
\hline \multirow{3}{*}{$\begin{array}{l}\text { Production } \\
\text { équivalente } \\
\text { agneaux (kg/UMOr) }\end{array}$} & $3^{\text {sme }}$ quartile & 13729 & 10827 & 8216 & 5896 \\
\hline & Moyenne & 11203 & 8403 & 6107 & 4128 \\
\hline & $1^{\text {er }}$ quartile & 8503 & 5575 & 4081 & 2069 \\
\hline
\end{tabular}


Tableau 6. Capital d'exploitation hors foncier selon les filières. Source : Réseaux d'élevage 2009.

\begin{tabular}{|l|c|c|c|}
\hline & €lUMOr & \multicolumn{2}{c|}{ €lunité de productivité physique } \\
\hline Exploitations laitières bovines & 221000 & \multicolumn{2}{|c|}{$1,0 / \mathrm{L}$ de lait } \\
\hline Exploitations laitières ovines & 209000 & $3,7 / \mathrm{L}$ de lait \\
\hline Exploitations laitières caprines & 145000 & \multicolumn{2}{|c|}{$1,1 / \mathrm{L}$ de lait } \\
\hline Exploitations allaitantes bovines & 319000 & \multicolumn{2}{|c|}{$10,9 / \mathrm{kg}$ de viande vive } \\
\hline Exploitations allaitantes ovines & 146000 & 18,1 & leq. $\mathrm{kg}$ carcasse d'agneaux \\
\hline
\end{tabular}

mances économiques tant au niveau de la valeur ajoutée nette que du revenu.

L'accroissement du capital est relativement progressif au fil des années, la capacité à le transmettre est un point névralgique pour les installations, notamment pour celles qui sont hors cadre familial.

Aujourd'hui, les exploitations qui enregistrent les plus fortes productivités du travail ont les montants de capitaux par unité de main-d'œuvre les plus élevés dans toutes les filières (par exemple pour les exploitations allaitantes bovines de plaine, le montant du capital représente $230 \mathrm{k} € / \mathrm{UMOr}$ pour la productivité du travail la plus faible contre $430 \mathrm{k} €$ pour la plus forte). Dans un contexte de volatilité des revenus, le besoin de sécurité financière au moment de l'installation, pour faire face à une mauvaise conjoncture, ne fait qu'accrô̂tre les besoins d'autofinancement.

\section{Conclusion}

Quelles conséquences de l'augmentation de la productivité physique du travail sur l'économie des exploitations?

Au plan méthodologique, nous nous sommes attachés dans cet article à clarifier la notion de productivité physique $\mathrm{du}$ travail. Cependant, le travail reste une donnée difficile à mesurer en agriculture en raison de son caractère familial. Nous avons utilisé les UMO pour l'évaluer (quantification du temps de travail «en équivalent temps plein») en étant conscients que les variations de temps de travail effectif pour une même UMO peuvent être considérables. Des enquêtes avec des méthodes de reconstitution analytique visant une quantification en heures permettraient d'identifier ces variations et de mesurer la productivité de la journée ou de l'heure de travail. Quant à la productivité économique du travail, nous l'avons mesurée par la valeur ajoutée rapportée à la main-d'œuvre, méthode classiquement utilisée par les économistes. Ces inves- tigations restent à poursuivre, par exemple l'évaluation des conséquences économiques d'un appel à l'entreprise ou au salariat partagé, comparé à celui d'un investissement propre.

L'analyse rétrospective montre que la productivité du travail en agriculture s'est accrue à un rythme beaucoup plus rapide que dans les autres secteurs économiques. Le volume produit et la valeur ajoutée brute en volume ramenés par actif ont connu un accroissement spectaculaire $(+400 \%$ en 30 ans pour la valeur ajoutée brute en volume). Cette évolution s'est faite de pair avec l'augmentation de la part du capital fixe et des consommations intermédiaires dans la valeur de la production, alors que les prix de ces dernières diminuaient moins vite en termes réels que celui des produits agricoles. Ainsi, la valeur ajoutée de la branche agricole a fortement diminué (en monnaie constante) depuis le milieu des années 1970. Le revenu agricole par actif ne s'est maintenu que grâce à l'accroissement des subventions et à la diminution du nombre d'actifs.

L'analyse de la productivité physique du travail et des principaux indicateurs économiques réalisée sur l'année 2009 pour les filières d'élevage met en exergue trois points déterminants :

- les écarts de productivité importants entre systèmes d'une même filière,

- l'importance de l'efficience économique du système (mesurée par le ratio $\mathrm{EBE} / \mathrm{PB}$ ) indicatrice de la recherche de cohérence du système de production et de l'optimisation des moyens de production,

- le poids de la conjoncture : si revenu et productivité physique évoluent souvent dans le même sens, la relation devient très ténue les années où les prix de vente baissent fortement, du fait notamment du poids des charges fixes dans les structures les plus importantes.

Les conséquences de l'accroissement de la productivité du travail doivent également s'analyser au-delà du revenu. En effet, dans toutes les filières d'élevage, son augmentation va de pair avec celle $\mathrm{du}$ capital, acquis progressivement et dont la transmission est un point névralgique de la vie de l'exploitation. Le niveau de capital requis, mis en regard du revenu espéré, de plus en plus volatil, peut être dissuasif. Une cessation d'activité génère un nouvel agrandissement des exploitations les plus proches et les plus à même de financer une augmentation de capital. En prolongeant la tendance actuelle (1 remplacement pour 5 départs, «départs précoces» inclus), le nombre d'exploitations bovines laitières pourrait se situer en 2014 entre 68 et 54000 (Perrot 2010) avec, pour conséquence, une pression accrue vers l'accroissement de la productivité de la main-d'œuvre.

Si l'on peut considérer que l'augmentation de la productivité du travail constitue une voie possible d'amélioration d'un système d'exploitation, il est cependant souhaitable qu'elle ne conduise pas à l'artificialisation de son fonctionnement sur les plans agronomique, zootechnique, économique ou ne le rende pas de plus en plus dépendant de ressources financières extérieures. Les effets, souvent peu perceptibles immédiatement, peuvent néanmoins se révéler très impactants à plus ou moins long terme (perte d'autonomie avec recours de plus en plus important aux intrants) ou dans un contexte économique et financier très volatil (qui semble devoir se généraliser). L'avenir n'est pas à un modèle unique d'exploitation (petites, moyennes ou grandes entreprises agricoles, système de production intensif ou extensif...), mais plutôt aux agriculteurs attentifs à une gestion de leur exploitation privilégiant la recherche permanente d'équilibre entre viabilité, vivabilité et transmissibilité (AFOCG01 2007, InterAFOCG 2007, Levallois 2010). Si beaucoup ont misé et misent encore sur l'agrandissement, la spécialisation et la rationalisation des tâches afin d'accroître la productivité physique de leur travail, d'autres optent pour la diversification et/ou la création de valeur ajoutée au travers d'une meilleure valorisation de leurs produits ou de la mise en œuvre de processus plus économes en intrants et en équipements. Ces stratégies ne sont ni cloisonnées ni exclusives ; la transformation et la commercialisation de produits agricoles concernent parfois les grandes exploitations et les petites peuvent être spécialisées ${ }^{5}$.

Des systèmes de production revenant aux bases de l'agronomie (complémentarité des productions, rotations de cultures privilégiant la prise en compte des

\footnotetext{
5 Sur ce thème, l'Institut de l'Elevage, l'INRA et l'inter AFOCG sont partenaires du projet CASDAR (2011 -2013) «Organisation, productivité du travail et sens du métier dans des élevages innovants».
} 
effets précédent/sensibilité du suivant, associations de cultures, autonomie alimentaire pour les animaux et les plantes), tels que les systèmes de polyculture élevage développés en agriculture biologique, se montrent autant, voire plus rémunérateurs que les systèmes conventionnels (Pavie et Lafeuille 2009a et b, Pôle AB Massif Central 2010). De la même manière, les systèmes herbagers économes qui valorisent au mieux le fonctionnement de l'écosystème (cultures pluriannuelles, associations prairiales à base de légumineuses, utilisation de l'effet précédent cultural, maximisation du pâturage...) et réduisent le recours à des intrants et à des équipements coûteux, se révèlent être intensifs du point de vue de la création de valeur ajoutée par hectare, performants en termes de productivité économique du travail et moins dépendants des aides publiques et des ressources fossiles (Garambois et Devienne 2010, Garambois 2011).

Si l'on sort de la France, on constate aujourd'hui à travers le monde une grande diversité de niveaux de productivité physique du travail suivant les systèmes de production (Agri benchmark 2011, IFCN 2011). Ne serait-ce qu'en Europe, une très large gamme peut être observée pour la production de lait de vache. L'analyse de l'évolution dans certains bassins laitiers de l'Europe du nord, notamment au Danemark où la productivité physique du travail est la plus élevée au monde, avec plus de $500 \mathrm{t}$ de lait/UMO, peut être riche en enseignements. Ces dernières années le phénomène de volatilité des revenus y a été encore plus fort que celui que nous avons connu en France. L'accroissement de la productivité se traduit en effet par «un besoin en capital toujours plus important, nécessitant un recours à des instruments financiers sophistiqués, censés permettre une gestion dynamique de l'endettement de ces exploitations» (Perrot et al 2011). L'éclatement des bulles foncières et financières a mis en lumière la fragilité financière d'une production laitière hautement capitalistique : le revenu moyen des exploitations laitières, qui avait atteint près de $40 \mathrm{k} € / \mathrm{UMO}$ en 2007 , est devenu négatif en 2008 et en 2009 (respectivement - $20 \mathrm{k} € /$ UMO et - $40 \mathrm{k} €$ ) et est resté nul en 2010. Les dynamiques de développement basées sur l'accroissement de la productivité physique du travail se sont traduites par une agriculture de moins en moins familiale au profit d'un modèle entrepreneurial dont les capitaux, très importants par actif, sont de plus en plus détenus par des structures financières.

De plus, dans les territoires où le foncier est limitant, par exemple aux PaysBas, il est difficile de concilier au niveau des systèmes de production l'accroissement de la productivité physique $\mathrm{du}$ travail et de bonnes performances environnementales.

En France, la productivité physique $\mathrm{du}$ travail reste encore faible (180 t de lait/UMO en 2010) au regard de ces pays, mais s'accroît régulièrement. Le développement de nouveaux moyens techniques (notamment automatisation et élevage de précision) laisse à penser que ce mouvement pourrait se poursuivre. Pour la filière lait de vache, la fin des quotas laitiers se traduira-t-elle par un nouvel accroissement de productivité dans le sillage des pays de l'Europe du Nord? Comment les projets de contractualisation vont-ils orienter la production ? Le mode d'attribution des aides peut avoir autant d'incidence sur les stratégies d'évolution des agricul- teurs que leur seul montant ; selon certains auteurs, les soutiens directs au revenu seraient sécurisants pour les agriculteurs soumis aux aléas de prix (OCDE 2011) et n'encourageraient pas forcément l'investissement (Sckohai et Moro 2009) ; d'autres les présentent comme un outil coûteux et inefficace pour protéger les agriculteurs contre la volatilité des prix des produits agricoles et des moyens de production (Kroll 2010). Les politiques publiques conforteront-elles cette tendance à l'accroissement de la productivité physique du travail ? Comment se répartiront les gains de productivité entre les différents acteurs de la branche (producteurs, industries agro-alimentaires, com-merce et consommateurs) ? Quelle sera l'attractivité du métier d'agriculteur? Enfin quelles seront les demandes sociétales de demain?

Quoi qu'il en soit, les systèmes de production et les agriculteurs continueront d'évoluer et de s'adapter ; dans tous les cas, la poursuite de l'analyse de la diversité des performances des exploitations, notamment pour les systèmes émergents en France et à l'étranger, du point de vue de la productivité physique mais aussi économique du travail, sera utile pour l'ensemble des filières d'élevage.

\section{Remerciements}

Nous remercions les éleveurs qui participent au dispositif des Réseaux d’Elevage, les ingénieurs départementaux qui assurent le suivi et l'enregistrement des données des exploitations et les ingénieurs qui animent les équipes régionales.

\section{Références}

AFOCG 01, 2007. 14 solutions pour soulager le travail sur les fermes : des agriculteurs témoignent. AFOCG de l'Ain, Bourg-enBresse, France, 40p.

Agri benchmark, 2011. Beef and sheep report, http://www.agribenchmark.org beef results beef sheep reports.html, 86p.

Bagoulla C., Chevassus-Lozza E., Daniel K., Gaigné C., 2010. Regional production adjustment to import competition: evidence from the French agro-industry. Am. J. Agr. Econ., 92, 1040-1050.

Bébin D., Lherm M., Liénard G., 1995. Quels résultats techniques et économiques en grands troupeaux de vaches allaitantes ? Le cas du Charolais. INRA Prod. Anim., 8, 213-225.

Benoit M., Laignel G., 2006. Méthodologie d'élaboration des résultats technico-écono- miques en élevage ovin allaitant. Illustration en France, en zone de plaine et de montagne. Options Méditerranéennes, série A, 70, 57-65.

Bourgeois L., Desriers M., 2002. Une concentration géographique des productions modérée depuis 1970. Agreste Cah., 2, 23-29.

Braibant M., 2007. Le partage volume prix Base 2000. INSEE notes méthodologiques, 119 p.

Butault J.P., 2004. Les soutiens à l'agriculture. Théorie, histoire, mesure. Editions Quae, Paris, France, 307p.

Butault J.P., 2008. La relation entre prix agricoles et prix alimentaires. Revue française d'économie. 23, 2, 215-241.

Butault J.P., Delame N., Rousselle J.M., 1995. Formation et répartition des gains de productivité dans l'agriculture française, analy- se par produit. Cah. Econ. Sociol. Rurales, 33, 55-72.

Charroin T., Ferrand M., 2010. Elaboration d'un jeu de coefficients pour analyser les coûts de structure d'une exploitation. Application aux charges de mécanisation des systèmes de polyculture-élevage. Renc. Rech. Rum., 17, 413-416.

Cochet H., Devienne S., 2006. Fonctionnement et performances économiques des systèmes de production agricole : une démarche à l'échelle régionale. Cah. Agric., 15, 578583.

Dedieu B., Servière G., Chauvat S., Tchakerian E., 2000. Bilan Travail pour l'étude du fonctionnement des exploitations d'élevage. Méthode. Coll. Lignes. Institut de l'Elevage, INRA, (Eds). 27p. 
Delaveau A., Chapelle P., Perrot C., Tchakérian E., Véron J., 1999. La cohérence des techniques fait le résultat économique. Les enseignements de l'approche globale appliquée aux exploitations d'élevage. Renc. Rech. Rum., 6, 3-10.

Desriers M., 2007. L'agriculture française depuis cinquante ans : des petites exploitations familiales aux droits à paiement unique. In : L'agriculture, nouveaux défis, coll. Réf., INSEE, Paris, France, 17-30.

Desriers M., 2011. Recensement agricole 2010. Structure des exploitations. Les productions se concentrent dans les exploitations spécialisées. Agreste Primeur, 272, 4p.

Dumont R., 1954. Progrès fourragers dans les Pays Scandinaves et en Grande-Bretagne. Collection Travail et Productivité, Editions SADEP, Paris, France, 111p.

Garambois N., 2011. Des prairies et des hommes. Les systèmes herbagers économes du Bocage Poitevin : agro-écologie, création de richesse et emploi en élevage bovin. Thèse AgroParisTech.

Garambois N., Devienne S., 2010. Evaluation économique, du point de vue de la collectivité, des systèmes bovins laitiers herbagers. Renc. Rech. Rum., 17, 25-32.

Giroux G., 2011. Recensement agricole 2010. Premières tendances. Agreste Primeur, 266, 4p.

Guihard V., Lesdos C., 2007. L'agriculture sur trente ans: une analyse comparative avec l'industrie et les services. In : L'agriculture, nouveaux défis, coll. Réf., INSEE, Paris, France, 47-63.

Guesdon J.C., 1985. Parlons vaches... Lait et viande en France, aspects économiques et régionaux. Paris, ITEB, Editions L'Harmattan, Paris, France, 146p.

IFCN, 2011. Dairy report 2011. For a better understanding of milk production world-wide. International Farm Comparison Network (Ed), 206 p.

Institut de 1'Elevage, 2010a. Calculer le coût de production en élevage bovins viande. Méthode nationale développée par l'Institut de l'Elevage dans le cadre du dispositif réseaux d'élevage. Réseaux d'élevage pour le conseil et la prospective, Coll. Théma, $8 \mathrm{p}$

Institut de 1'Elevage, 2010b. Résultats 2008 et estimation 2009 pour les exploitations bovins lait. Synthèse annuelle des Réseaux d'Elevage. Coll. Résultats annuels, 42p.

Institut de 1'Elevage, 2011a. L'élevage bovin allaitant français à l'horizon 2015 et perspecti- ves 2035. Coll. Le dossier Economie de l'Elevage, 416, 48p.

Institut de 1'Elevage, 2011b. Résultats 2009 et estimation 2010 pour les exploitations bovins lait. Synthèse annuelle des Réseaux d'Elevage. Coll. Résultats annuels, 52p.

InterAFOCG, 2007. Organiser son travail et gérer son temps sur les fermes. Guide pour l'accompagnement. Educagri Editions, Dijon, France, 92p.

Kroll J.C., 2010. La PAC en discussion. Un nouvel outil pour une nouvelle approche. Écon. Rurale, 318-319, 123-126.

Lacroix A., Mollard A., 1991. Mesurer le travail agricole : de l'enregistrement à la reconstitution analytique. Cah. Econ. Sociol. Rurale, 20, 20p.

Lebrun V., 1983. Une méthode d'étude du système de production au niveau de l'exploitation, ITEB, Paris, France, 64p.

Levallois R., 2010. Gestion de l'entreprise agricole. De la théorie à la pratique. Presses de l'Université. Laval, France, 386p.

Lherm M., Bébin D., Liénard G., 1983 Evolution récente des systèmes de production en troupeaux allaitants charolais des zones herbagères. Situation 1981 d'un groupe d'élevages de la Nièvre et de la Creuse. Evolution par rapport à 1980, 1979, 1975 et 1971. Bull. Tech., CRZV, Theix, France, 51, 63-83.

Mabile S., 1990. La productivité en France de 1970 à 1989: une approche sectorielle. Econ. stat., 237-238, 69-86.

Malassis L., 1969. Agriculture et croissance économique. Écon. rurale, 79-80, 19-32.

Mazoyer M., Roudart L., 1997. Histoire des agricultures du Monde. Edition Seuil, Paris, France, $534 \mathrm{p}$.

Mounier A., 1992. Les théories économiques de la croissance agricole. INRA Economica, Paris, France, $427 \mathrm{p}$

OCDE, 2011. Managing risk in agriculture: policy assessment and design. OECD publishing, Paris, France, 255p

Pavie J., Lafeuille O., 2009a. Valorisation des données technico-économiques des exploitations allaitantes biologiques suivies dans les réseaux d'élevage. Evolutions d'un échantillon constant sur 7 campagnes (2000-2006). Réseaux d'élevage pour le conseil et la prospective, collection références. Institut de l'Elevage, Paris, France, 40p.

Pavie J., Lafeuille O., 2009b. Valorisation des données technico-économiques des exploitations laitières biologiques suivies dans les réseaux d'élevage. Evolutions d'un échantillon constant sur 7 campagnes (2000-2006). Réseaux d'élevage pour le conseil et la prospective, collection références. Institut de l'Elevage, Paris, France, 36p.

Perez C., 2009. Technological revolutions and techno-economic paradigms. Camb. J. Econ., 34, 185-202.

Perrot C., 2010. Projection du nombre d'exploitations bovines laitières ou allaitantes françaises. Une approche par modélisation démographique intégrant des effets territoriaux. Renc. Rech. Rum., 211-214.

Perrot C., Mottet A., You G., 2011. Les modèles laitiers européens du nord de l'UE à l'épreuve de la volatilité. Renc. Rech. Rum., $18,317-320$

Pochon A., 2002. La prairie temporaire à base de trèfle blanc, 3 ème édition mise à jour Cedapa (Ed), 127p.

Pochon A., 2008. Agronomes et paysans, un dialogue fructueux. Editions Quae, Versailles, France, 72p

Pôle Agriculture Biologique Massif Central, 2010. Analyse du fonctionnement et des performances des systèmes d'élevages agrobiologiques du Massif Central. Résultats de la campagne 2008. Edition 2010. Pôle AB Massif Central, ABioDoc (Eds), 83p.

Sckokai P., Moro D., 2009. Modeling the impact of the CAP Single Farm Payment on farm investment and output. Eur. Rev. Agric. Econ., 36, 395-423.

Sébillotte J., 1966. Ebauche de classification économique des exploitations agricoles françaises en 1963. Econ. Rurale, 70, 43-54.

Veysset P., Lherm M., Bébin D., 2005a. PAC, prix et primes : quelles incidences sur les types de produits vendus en élevage bovin allaitant Charolais ? Renc. Rech. Rum., 12, 35.

Veysset P., Lherm M., Bébin D., 2005b. Evolutions, dispersions et déterminants du revenu en élevage bovin allaitant charolais. Etude sur 15 ans (1989-2003) à partir d'un échantillon constant de 69 exploitations. INRA Prod. Anim., 18, 265-275.

Veysset P., Sarzeaud P., Broutard J., Carteron P., Ingrand S., 2008. Transmission des exploitations en élevage bovin allaitant : problématiques de la fin de carrière et leviers de réussite pour l'installation. Renc. Rech. Rum., 15 , 247-250.

Voisin A., 1957. Productivité de l'herbe, La maison rustique, Paris, France, 467p.

\section{Résumé}

De tous les secteurs de l'économie, c'est l'agriculture qui a connu l'accroissement le plus rapide de la productivité du travail au cours des cinquante dernières années. La production en volume de la branche a été multipliée par 2,2 entre 1955 et 2010, grâce à l'utilisation croissante des intrants et à la mobilisation d'un capital toujours plus important (matériel et bâtiments). Sur cette même période, la population active agricole est passée de 31 à 3,4\% de l'emploi total. Les subventions jouent aujourd'hui un rôle déterminant dans le maintien du revenu agricole par actif. L'analyse de la productivité du travail pour les filières bovine, ovine et caprine montre que l'accroissement de la productivité physique et des principaux indicateurs économiques ne vont pas forcément de pair. Il existe des écarts de productivité physique du travail importants entre systèmes et entre filières. Si, en bonne conjoncture de prix, les systèmes à haute productivité physique du travail dégagent les meilleurs revenus, une évolution défavorable des prix induit pour ces systèmes une fragilisation économique révélant ainsi leur faible capacité de résilience. La recherche de cohérence du système de production et de l'optimisation des moyens de production apparaît toujours comme un élément déterminant des performances économiques. L'orientation des exploitations sera largement induite par la répartition des gains de productivité entre les différents acteurs de la branche (producteurs, industries agricoles et alimentaires, commerce et consommateurs) ainsi que par les choix des pouvoirs publics. 


\begin{abstract}
Labour productivity and economy in herbivore rearing: concepts, analysis and stakes

Labour productivity in agriculture has grown faster than the other sectors of the economy over the last fifty years. Volume production of the branch was multiplied by 2.2 from 1955 to 2010, thanks to the increasing use of input and a more important mobilization of capital (equipment and buildings). At the same time the agricultural labour force decreased from 31 to $3.4 \%$ of total employment. Today, subsidies have a decisive role in the upholding of farm income per worker. The analysis of labour productivity for cattle, sheep and goat productions shows that the economic indicators are not necessarily linked to an increase in physical productivity. There are differences in physical productivity of labour between systems and production sectors. In a favourable economic situation, systems with high physical productivity of labour get the best income, but when the economic situation becomes unfavorable these systems appear financially weak and reveal their low resilience capability. The consistency of the production system and the optimization of means of production always appear as a determinant of economic performance. The distribution of productivity gain between the different actors in the sector (producers, agricultural and food industries, trade and consumer) and the choice of government will remain decisive for farm orientation.
\end{abstract}

CHARROIN T., P. VEYSSET P., DEVIENNE S., FROMONT J.-L., PALAZON R., FERRAND M., 2012. Productivité du travail et économie en élevages d'herbivores : Définition des concepts, analyse et enjeux. In : Numéro spécial, Travail en élevage. Hostiou N., Dedieu B., Baumont R. (Eds). INRA Prod. Anim., 25, 193-210. 